OPEN ACCESS

Edited by:

Genovefa Kefalidou, University of Leicester,

United Kingdom

Reviewed by:

Alexandros Rouchitsas,

Luleå University of Technology,

Sweden

Amir Rasouli,

Huawei Technologies, Canada

*Correspondence:

Andreas Riegler

andreas.riegler@fh-hagenberg.at

Specialty section:

This article was submitted to

Digital Impacts,

a section of the journal

Frontiers in Human Dynamics

Received: 01 April 2021

Accepted: 30 July 2021

Published: 12 August 2021

Citation:

Riegler A, Riener A and Holzmann C (2021) A Systematic Review of Virtual

Reality Applications for Automated

Driving: 2009-2020.

Front. Hum. Dyn 3:689856.

doi: 10.3389/fhumd.2021.689856

\section{A Systematic Review of Virtual Reality Applications for Automated Driving: 2009-2020}

\author{
Andreas Riegler ${ }^{1,2,3 *}$, Andreas Riener ${ }^{2}$ and Clemens Holzmann ${ }^{1}$ \\ ${ }^{1}$ University of Applied Sciences Upper Austria, Hagenberg im Mühlkreis, Mühlkreis, Austria, ${ }^{2}$ Technische Hochschule Ingolstadt, \\ Ingolstadt, Germany, ${ }^{3}$ Johannes Kepler University Linz, Linz, Austria
}

While virtual reality (VR) interfaces have been researched extensively over the last decades, studies on their application in vehicles have only recently advanced. In this paper, we systematically review 12 years of VR research in the context of automated driving (AD), from 2009 to 2020. Due to the multitude of possibilities for studies with regard to VR technology, at present, the pool of findings is heterogeneous and non-transparent. We investigated $N=176$ scientific papers of relevant journals and conferences with the goal to analyze the status quo of existing VR studies in AD, and to classify the related literature into application areas. We provide insights into the utilization of VR technology which is applicable at specific level of vehicle automation and for different users (drivers, passengers, pedestrians) and tasks. Results show that most studies focused on designing automotive experiences in VR, safety aspects, and vulnerable road users. Trust, simulator and motion sickness, and external human-machine interfaces (eHMls) also marked a significant portion of the published papers, however a wide range of different parameters was investigated by researchers. Finally, we discuss a set of open challenges, and give recommendation for future research in automated driving at the VR side of the reality-virtuality continuum.

Keywords: virtual reality, automated driving, systematic review, user studies, augmented reality, user experience, usability, classifications

\section{INTRODUCTION}

The advancement of automated driving (AD) systems and corresponding human-machine interfaces (HMI) marks currently one of the biggest transformations in transportation research and development. In 2018, the Society of Automotive Engineers (SAE On-Road Automated Vehicle Standards Committee, 2021) published the latest version of their definition of vehicle automation, and their different levels, addressing challenges and laying the foundation for future standardization, as well as establishing a common language. With SAE Level 2 (L2) driving automation already on the road, it is only a matter of time until SAE Level 3 (L3) automated vehicles, or even higher levels, become commercially available. In the automotive domain specifically, the progress of both automated driving and mixed reality technology are pushing academia and industry to explore new vehicular user interface concepts. More and more HMI concepts investigate the reality-virtuality (RV) continuum (Milgram et al., 1995), from conventional 2D screens towards augmented reality (AR), and virtual reality (VR). Application areas include automotive, aeronautics, logistics, production, education, cultural heritage, and others (Dey et al., 2018). In AR, digital virtual images are overlaid on the real world, such that the digital content is aligned with real world 


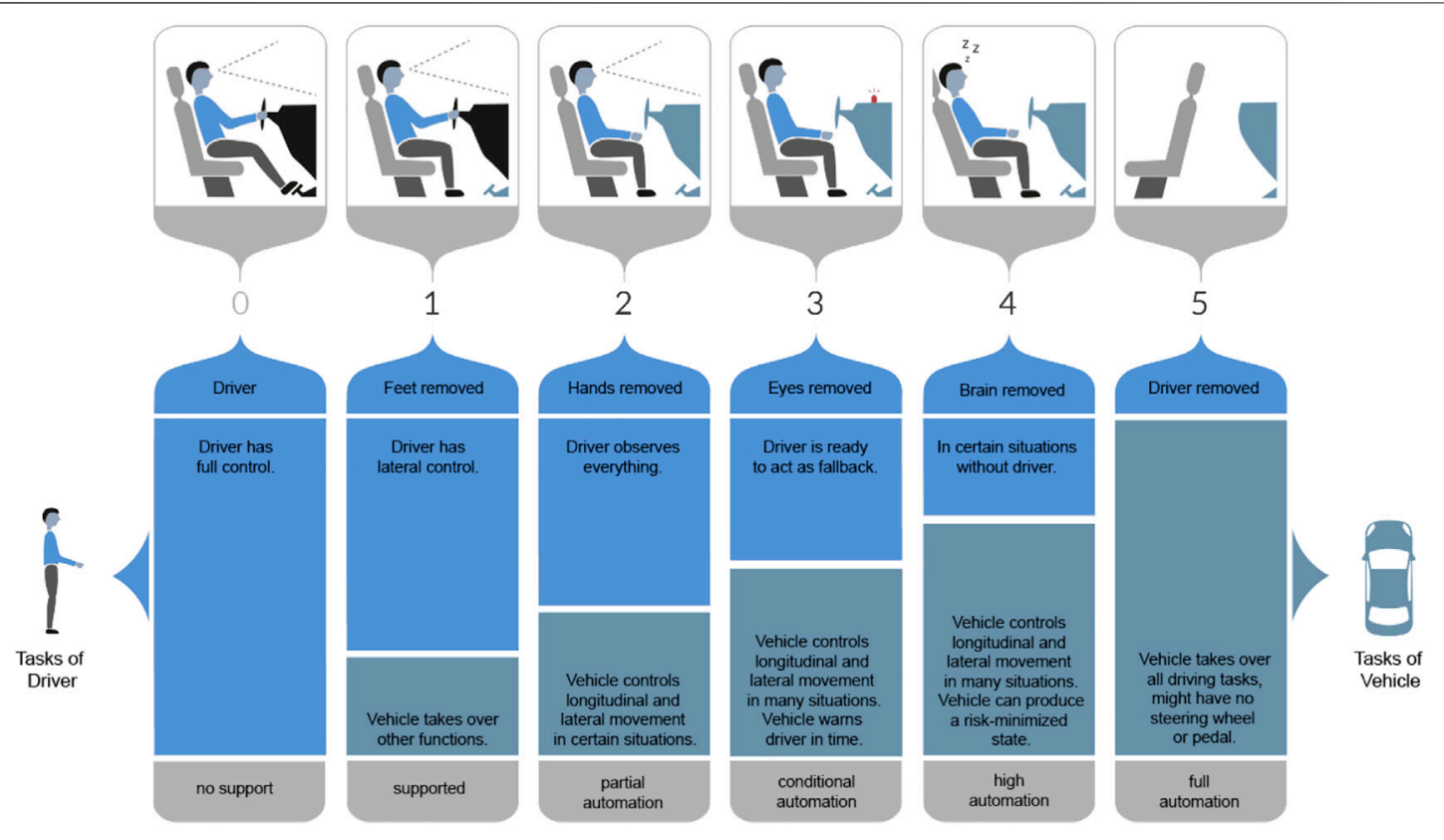

FIGURE 1 | SAE levels of vehicle automation (SAE On-Road Automated Vehicle Standards Committee, 2021).

objects (Azuma, 1997). Contrarily, in VR, environments solely consist of 3D digital objects (Steuer, 1992). Subsequently, mixed reality (MR) encompasses all potential positions on the RV continuum, i.e., between the real world and the virtual environment. Specifically, in the automotive domain, MR technology has been adopted for a variety of use cases. For example, head-up displays (HUDs) and larger windshield displays (WSDs) utilize AR technology to increase drivers' situational awareness by keeping their gaze on the road, and even going further by displaying work- or entertainment-related content for performing non-driving related tasks (NDRTs) for more highly automated vehicles in prototype studies.

\subsection{The Role of Virtual Reality in Driving Research}

VR can be categorized according to the level of immersion provided to the users. Non-immersive visualizations, such as desktop monitor setups are easy and cost-efficient to implement. Semi-immersive VR is accomplished with head-up displays (HUDs) that provide an augmentation of the real world with a digital overlay. In contrast, head-mounted displays (HMDs), and Cave automatic virtual environments (CAVEs), which are comprised of projection being cast on walls, provide a high level of immersion to the user, as the real environment is moving into the background.

The advancement of vehicle automation from manual and assisted driving towards highly and fully automated vehicles requires a classification for these different automation levels, as mixed traffic, where manual and automated vehicles will share the road, will be more and more common. Therefore, the SAE On-Road Automated Vehicle Standards Committee
(2021) has introduced a common language to identify these automation levels (see Figure 1). Each level of vehicle automation provides both opportunities and challenges for researchers and practitioners. While for the lower levels, situation awareness and safety concerns need to be addressed, higher automation levels could bring new interaction and passenger experiences to the car.

Consequently, VR technology has been used to research automated driving features and experiences, while remaining in a safe and controlled environment. Additionally, the current limited availability of highly automated vehicles drives researchers to investigate $\mathrm{AD}$ scenarios by other means, such as virtual reality simulations. Furthermore, technological advancements and increased affordability of VR equipment facilitate its utilization. Studies show that participants' subjective and psychological responses in virtual reality environments are closely coupled to their experience and behaviors in a real-world setup (Slater et al., 2006). Additionally, VR setups allow for controlled, reproducible, and safe experiments (De Winter et al., 2012). Furthermore, data collection (e.g., driving performance) is less cumbersome than with real vehicles (De Winter et al., 2012). Pettersson et al. (2019) compared a VR setup to a field study, and found that while VR studies do not fully replace field studies, they can provide added value to driving simulators and desktop testing. In particular, VR can be used for conveying the overall principles, or "the story" behind a user study. Another comparison study conducted by Langlois et al. (2016) revealed that the virtual environment is able to replicate relative distance perception between the virtual items (e.g., vehicles, pedestrians, road objects) and their overlays. Therefore, Langlois et al. (2016) recommend that user experiments can be conducted in virtual environments with 
simulated AR HUDs or WSDs. Further comparison studies (e.g., Weidner et al., 2017; Bolder et al., 2018; Rhiu et al., 2020) also show the viability and applicability of using VR for driving research.

Additionally, VR technology for driving research is not only used as a "means to an end" (i.e., to conduct user studies), but increasingly, the use of VR HMDs for drivers/passengers is also being explored. As our intention of this survey is to give a holistic overview of user studies conducted with VR in the automotive domain, we include both aspects.

As a result, there is a wide range of challenges that need to be overcome, and a multitude of papers addressing these timely issues have been published over the last years. However, it is often hard to integrate and/or compare the obtained findings, as topics are often investigated differently. Furthermore, due to the sheer amount of results, it is hard for researchers to identify gaps where they could build upon. Thus, we claim that the time has come to systematically review which topics in research on virtual reality applications in driving automation received the most attention, and which methods have been applied to investigate these. To help the automotive UI community improve usability and user experience of VR applications, this paper provides an overview of 12 years of VR studies carried out in the automotive domain. We chose to start with the year 2009, as this was the year the AutomotiveUI community (AutomotiveUI, 2009) was established, with particular focus on user interface design and HCI research in the automotive domain. The overarching aim of this paper is to provide an overview of current and past use of VR in automated vehicles, with respect to their level of vehicle automation, to support researchers and practitioners finding example papers encompassing related studies, and to help determine current research gaps that should be filled with future studies. Hence, this survey holds a mirror to the automotive UI community on what has been accomplished and which future aspects need more attention.

\subsection{Topics of Interest}

The focus of this survey is on the combination of two emerging technologies: 1) virtual reality applications and usage, and 2) automated driving systems. Gartner's hype cycle (Gartner, Inc., 2021), an indicator for common patterns that occur with emerging technologies, visualizes the current maturity status of these technologies which is an estimator for growth or depletion of a given technology. In 2014, both augmented and virtual reality were in the phase of "Trough of Disillusionment", indicating that these technologies started to be rigorously tested with experiments and implementations (Gartner, Inc., 2014). Five years later, both AR and VR were removed from the Gartner hype cycle for emerging technologies (Gartner, Inc., 2019), meaning that they left the experimental status, and had matured, i.e., become usable and useful. Autonomous driving at SAE level 4 has not yet reached this status yet. The implication for automotive $\mathrm{HCI}$ researchers and practitioners is that MR will enter conditionally automated vehicles (AVs) in the near term. Accordingly, Gabbard et al. (2014) state that manufacturers are expected to build upon these technologies and provide AR applications in the foreseeable future. For example, Daimler has recently previewed an AR HUD which enables the visualization of world-relative navigational cues and vehiclerelated information to the driver (Daimler, 2020). In the meantime, VR technology is used to simulate AR applications, as technical and cost constraints have not yet enabled a wide adoption of pure AR systems (e.g., Riegler et al., 2019b; Gerber et al., 2019). We therefore want to review the role that virtuality plays, and inherently investigate the VR side of the RV continuum (Milgram et al., 1995).

We also want to reflect that MR technology could be viewed as a "means to an end", that is to investigate how this technology can be utilized to calibrate trust and acceptance in automated driving. Therefore, another important topic we intend to evaluate are application areas. We are interested in the evolution of early VR applications for non-automated vehicles (up to SAE L2) towards futuristic concepts and studies for fully automated vehicles (SAE L5). This includes route navigation, system status feedback, performing work- and entertainment related activities, among others. For example, early research efforts in automated driving have often focused on take-over request (TOR) scenarios, where the vehicle exceeds its operational design domain or encounters an emergency, and prompts the driver to interrupt their non-driving related task (NDRT), and to transition to manual vehicle control. VR application research therefore aims at investigating underlying mechanisms needed to substitute former driving experiences with other, potentially meaningful activities, and appropriate user interfaces which carve out advantages and balance negative effects of automation. With increasing vehicle automation, vehicles will transform more and more into mobile work and living spaces (Schartmüller et al., 2018).

As automated driving systems advance, traditional drivers evolve into passive passengers. Consequently, another focus of this survey is on (potential) users of VR technology in and around vehicles, such as drivers, passengers, pedestrians, and other road users. New paradigms are needed for communicating intent between automated vehicles and pedestrians, for example using external human-machine interfaces (eHMIs). MR technology can be applied to investigate corresponding scenarios, e.g. using simulated environments. Hence, we provide an overview of utilized devices for research in virtual reality combined with automated driving. This includes headmounted displays (HMDs), HUDs, WSDs, cave automatic virtual environments (CAVEs), 2D monitor setups and projections, among others.

\subsection{Previous Literature Reviews and User Study Survey Papers}

In contrast to the recent survey on user studies on automated driving conducted by Frison et al. (2020), this work focuses on user studies on vehicles with different levels of driving automation conducted within virtual reality environments. In 2020, Riegler et al. (2020b) presented workshop results on grand challenges in mixed reality applications for intelligent vehicles, and thus provides a good overview for future user studies in the form of a research agenda, whereas our view is more focused on 
VR as a subtopic of mixed reality, and covers both the main research themes of recent user studies and how these studies were conducted. Gabbard et al. (2014) focus on driver challenges and opportunities for in-vehicle AR applications. We recognize that VR should also receive attention for in-vehicle applications, both by using VR as a tool to conduct user studies, and as an end product (e.g., HMDs). McGill and Brewster (2019), in their review only look at user studies focussing on passenger experiences with VR HMDs, while we take a holistic approach towards VR usage for driving-related user studies. Another subtopic of VR in AD research was investigated by Rangelova and Andre (2019) in their survey on simulation sickness caused by VR HMDs. In the present literature review, we provide an overview of all potential VR devices causing simulator sickness, including HMDs and CAVEs, for example. Additionally, Rouchitsas and Alm (2019) provide a review of external human-machine interface (eHMI) research for vehiclepedestrian communication, both in VR and non-VR setups. However, their focus was on topics covered by the reviewed user studies, while we emphasize methodological approaches, used measures and VR devices, as well as discuss implications and potential future eHMI research directions. This wealth of recent surveys in all these adjacent areas emphasizes the importance and timeliness of the topic, in our opinion.

\subsection{Novelty and Contribution}

To capture the latest trends in VR in $\mathrm{AD}$ research, we have conducted a thorough, systematic review of 12 years of relevant papers published between 2009 and 2020. We classified these papers based on their application areas, methodologies used, and their utilized levels of vehicle automation. Moreover, we intend to provide impetus towards a standardization of methodology concerning self-report, behavioral, and physiological measures in VR driving studies.

The main contribution of this work is four-fold, concerning the status quo and derivation of future research directions:

- Identify the primary application areas for automotive user interface research using VR technology.

- Provide an overview of the methodologies and VR environments commonly utilized in driving automation research.

- Identify open challenges and opportunities, and propose recommendations for the research of VR in $\mathrm{AD}$.

- Provide a set of recommendations and emerging possibilities for VR study design in driving automation research.

The rest of the paper is organized as follows: Section 2 details the method and process we followed to find, rate and review the relevant papers. Section 3 then gives a high-level overview of the core relevant papers and studies, and establishes the classifications, followed by detailed reports on each of the classifications. Section 4 provides details each of the classifications, highlighting some of the most impactful user studies as well as challenges for each application area. Section 5 concludes by summarizing the review and identifying challenges, opportunities, and recommendations for future research. Finally, in the appendix, we have included a list of all papers reviewed in each of the application areas.

\section{METHODOLOGY}

Considering the current research status, topics of interest in virtual reality $\mathrm{HCI}$ research in automated driving, and the authors' experience in VR and $\mathrm{AD}$ research, we defined the reviewing process for this literature review. For the analysis of related literature, we reviewed publications at the intersection of augmented and virtual reality, human-computer interaction, and automated driving.

We followed a process of three stages, which are search (find relevant candidate publications), rate (determine the relevance of all candidates) and code (extract important details of the core relevant papers) in our literature review. Although these steps were mainly conducted in sequence, we performed several reiterations and overlaps to ensure their validity.

Search: For exploring the body of work in VR in AD, we conducted a thorough literature research using the standard scientific search engines and databases ACM Digital Library, IEEE Xplore, Science Direct, Wiley Online Library, and Google Scholar. Furthermore, besides being a rich source of VR and AD research, they contain influential conferences and highly ranked journals such as CHI, DIS and TOCHI, VRST, AutomotiveUI, IV, and VR. We based our search on combinations of the terms "augmented reality", "virtual reality", "mixed reality", "driving”, "automated vehicle", and "vehicle automation":

“augmented reality" OR “virtual reality" OR "mixed reality"
AND
“driving" OR “automated vehicle" OR "vehicle automation"

Additionally, we focused our search on contributions integrating aspects of $\mathrm{VR}$ in $\mathrm{AD}$, which were introduced in top level AR/VR visualization, human-computer interaction, and automotive user interfaces conferences and journals. The basis for the selection process was research papers that were published in the respective venues between 2009 and up to 2020 (inclusive). The literature corpus also encompassed literature from a pre-existing list based on prior work by the authors. The initial determination of inclusion in the list of candidates was mainly based on their keywords, title, and abstract in order to initially reduce the number of papers in a reasonable way, while at the same time ensuring that no potentially relevant paper would be excluded. We further excluded the current year (2021), since the publication year was not yet finished and thus not all possibly relevant papers were available. In addition to papers found in this initial search, some papers were later rated as relevant, e.g., by analyzing the related work of reviewed publications. Finally, this stage resulted in a total of 293 candidate papers which were identified as interesting for further analysis.

Rate: For an initial classification of the found literature, the full set of 293 candidate papers was rated on their relevance for VR 


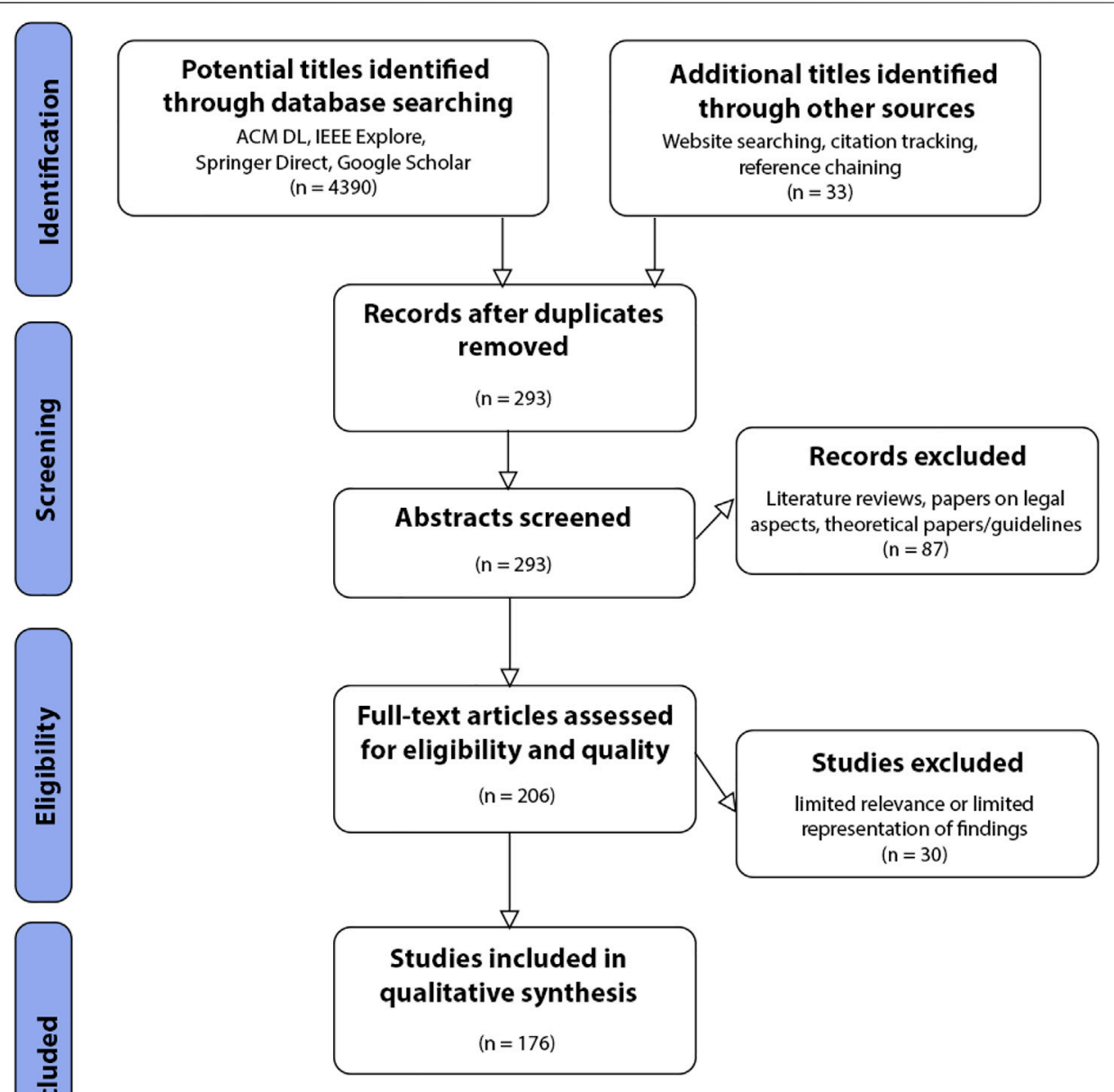

FIGURE 2 | PRISMA flow diagram depicting the paper selection process.

in $\mathrm{AD}$. Prior to the rating process, the authors discussed and established initial relevance criteria. Subsequent to determining this initial set of criteria, every paper was analyzed if it is interesting for further consideration in the list of core relevant papers. These continuous meetings during the rating stage also helped to further refine and substantiate the relevance criteria, and to unify the way in which they were used among the authors. For the entire literature corpus, the criteria for inclusion depended on quality and the provision of user study details. For example, we excluded publications where only an abstract or no detailed description were provided. Further criteria for relevance were based on the type of paper, such that for example papers covering pedestrian safety using augmented reality technology were rated relevant based on whether the system was considered to be utilized in the context of automated driving. Papers focusing on interaction techniques or devices, as well as user studies and other evaluations or benchmarks were considered with regard to the quality or usefulness of the particular interaction modality or study in the context of VR in AD. Furthermore, we disregarded literature reviews, as well as juridical, theoretical, or ethical papers (e.g., Inners and Kun, 2017). Out of the set of candidate papers, 176 core relevant papers were finally identified.

Code: After selecting the papers, we established a reviewing strategy for literature analysis aimed at developing a standardized reviewing procedure. We used the PRISMA flow diagram (Moher et al., 2010), shown in Figure 2, to decide in a standardized and step-wise manner whether to record the individual papers. Additionally, in several brainstorming sessions, we introduced a categorization of sub-topics of interest to be investigated. Based on the keywords and abstract of the respective papers, as well as recent and historic paper tracks at the AutomotiveUI conference, the "premier forum for UI research in the automotive domain" (AutomotiveUI, 2009), we categorized our literature corpus into twelve main application areas. These sub-topics are reflected by the sub-sections in Section 3. If a 
TABLE 1 | Summary of 176 reviewed papers.

\begin{tabular}{|c|c|c|c|c|c|c|c|c|c|c|}
\hline \multirow[t]{2}{*}{ Application area } & \multirow[t]{2}{*}{ Paper } & \multirow{2}{*}{$\begin{array}{c}\text { Mean author } \\
\text { Count }\end{array}$} & \multicolumn{2}{|c|}{ Publication } & \multicolumn{3}{|c|}{ Device } & \multicolumn{3}{|c|}{ Level of automation } \\
\hline & & & Journal & Conference & HMD & CAVE & Monitor & LO-2 & L3-4 & L5 \\
\hline Navigation \& Routing & 11 & 3.45 & 1 & 11 & 3 & 6 & 2 & 10 & 0 & 1 \\
\hline Driving Behavior & 18 & 4.00 & 9 & 9 & 8 & 8 & 3 & 15 & 3 & 0 \\
\hline Safety & 19 & 3.80 & 8 & 10 & 4 & 10 & 5 & 13 & 5 & 1 \\
\hline Education \& Training & 11 & 4.09 & 4 & 7 & 7 & 2 & 5 & 7 & 4 & 0 \\
\hline UI Design \& Prototyping & 26 & 4.38 & 9 & 17 & 6 & 11 & 9 & 14 & 6 & 6 \\
\hline Passenger Experiences & 12 & 4.92 & 1 & 11 & 8 & 2 & 3 & 1 & 2 & 9 \\
\hline Simulator Sickness & 8 & 3.00 & 2 & 6 & 7 & 1 & 0 & 3 & 1 & 4 \\
\hline Interaction Modalities & 16 & 3.88 & 4 & 12 & 4 & 5 & 8 & 9 & 6 & 1 \\
\hline Trust \& Acceptance & 12 & 4.42 & 5 & 7 & 7 & 3 & 2 & 3 & 0 & 9 \\
\hline External HMl & 15 & 4.53 & 6 & 9 & 14 & 0 & 1 & 0 & 0 & 15 \\
\hline Vulnerable Road Users & 20 & 4.30 & 7 & 13 & 12 & 6 & 3 & 8 & 0 & 12 \\
\hline Traffic Simulation & 8 & 3.75 & 3 & 5 & 2 & 0 & 7 & 6 & 1 & 1 \\
\hline Overall & 176 & 4.04 & 59 & 117 & 82 & 54 & 48 & 89 & 28 & 59 \\
\hline
\end{tabular}

paper covered multiple application areas (e.g., navigation and trust), we would determine its classification by the core intent of that paper. For example, if the effect of various navigational cues on driver trust and acceptance was investigated, we would classify that paper into the Trust, Acceptance, and Ethics application area (e.g., Wintersberger et al., 2017), rather than Vehicle Navigation and Routing. Following the categorization, we coded the core relevant papers using keywords derived from the analyzed papers, or based on already existing taxonomies on the respective topic. During the coding stage, we removed 35 non-referenced papers from the list of core relevant papers which we attribute to the evolving process of relevance assessment. Furthermore, an additional 20 papers were included to the list of core relevant papers in this stage of the process, as we deemed them to be relevant to a certain sub-topic.

\subsection{Limitations and Validity Concerns}

Although we made every effort to be methodical and rigorous in our selection and review of these 176 papers, we acknowledge several limitations and validity issues with our approach. First, we might have missed bibliographic sources. While the use of the aforementioned bibliographic databases has the advantage of including a variety of publication venues and topics, it is possible that we have overlooked venues and papers that should have been added. The second involves the used search parameters. Although the search terms we employed seem genuine and conscientiously selected, there may be papers that did not add the suggested keywords when describing AR, VR, or MR applications and usages in the automotive domain. For example, papers might have described driving behavior research evaluated with a VR driving simulator, yet, did not include VR in their keywords, title, or abstract. Finally, we did not measure the average citation count (ACC) for each paper. While this filter was applied in previous literature reviews (e.g., Dey et al., 2018), we refrained from excluding papers based on their impact, as some papers with seemingly narrow use cases, and maybe no immediately generalizable application area for VR in $\mathrm{AD}$ research, might soon prove influential.

\section{HIGH-LEVEL OVERVIEW OF REVIEWED PAPERS}

In total, we investigated the user studies reported in 176 papers. Table 1 provides a summary statistics for these papers, and Table 2 gives an overview of the user study details.

\subsection{User Studies Over Time}

Figure $3 \mathrm{~A}$ visualizes the total number of VR papers published between 2009 and 2020 with automotive context, categorized by papers at different stages of the RV continuum. The figure shows that the number of AR and VR papers published in 2020 is more than ten times that published in 2009. Overall, the number of papers utilizing mixed reality technology is growing rapidly.

\subsection{Study Design}

As displayed in Table 2, most of the papers (127, or $76 \%$ ) employed a within-subjects design, 29 papers (17\%) used a between-subjects design, and 12 papers (7\%) used a mixedfactorial design. For eight studies, it was not reported or clear from the study details which study design they applied.

\subsection{Study Type}

We found that it was uncommon for researchers to provide details on pilot studies before their main study. Only 21 papers (11\%) reported conducting a pilot study in their experimentation process. Only six papers report just the results of the pilot study, without further main studies, which indicates that the importance of pilot studies is not well acknowledged. Most studies (156, or $83 \%)$ were performed in controlled laboratory environments, while only 10 studies (6\%) were conducted in a natural environment or as a field study. In the Traffic Simulation application area, we found only two user studies were conducted, as simulations usually employ heuristics for evaluation. As most of the user studies were formal user studies with participants, and, with the exception of Traffic Simulation studies, employed no heuristic evaluation, might be an indication that heuristics and standardizations (Dey et al., 2018) need to be developed for future VR applications and use cases in the automotive domain. Due to design limitations, many 
TABLE 2 | Summary of the user studies reported by the 176 reviewed papers.

\begin{tabular}{|c|c|c|c|c|c|c|c|c|c|c|c|c|c|}
\hline \multirow[t]{2}{*}{ Application area } & \multicolumn{3}{|c|}{ Study type } & \multicolumn{3}{|c|}{ Study design } & \multicolumn{3}{|c|}{ Study location } & \multicolumn{3}{|c|}{ Data collected } & \multirow{2}{*}{$\frac{\text { Median }}{\text { Participants }}$} \\
\hline & Lab & Field & Pilot & Within & Between & Mixed & Indoor & Outdoor & Both & Quant. & Qual. & Both & \\
\hline Navigation \& Routing & 9 & 0 & 2 & 8 & 0 & 2 & 12 & 0 & 0 & 0 & 3 & 8 & 22 \\
\hline Driving Behavior & 15 & 2 & 1 & 15 & 2 & 1 & 16 & 2 & 0 & 9 & 1 & 8 & 24 \\
\hline Safety & 19 & 0 & 1 & 11 & 6 & 2 & 18 & 0 & 0 & 8 & 3 & 8 & 32 \\
\hline Education \& Training & 11 & 1 & 0 & 6 & 5 & 0 & 9 & 0 & 1 & 1 & 6 & 4 & 52 \\
\hline UI Design \& Prototyping & 24 & 0 & 7 & 18 & 4 & 4 & 23 & 2 & 1 & 4 & 4 & 18 & 25 \\
\hline Passenger Experiences & 9 & 3 & 3 & 9 & 1 & 1 & 10 & 2 & 0 & 0 & 9 & 3 & 20 \\
\hline Simulator Sickness & 5 & 3 & 0 & 7 & 1 & 0 & 5 & 3 & 0 & 1 & 3 & 4 & 28 \\
\hline Interaction Modalities & 16 & 0 & 2 & 15 & 1 & 0 & 16 & 0 & 0 & 1 & 1 & 14 & 20 \\
\hline Trust \& Acceptance & 12 & 0 & 1 & 8 & 4 & 0 & 12 & 0 & 0 & 0 & 6 & 6 & 28 \\
\hline External HMl & 15 & 0 & 2 & 13 & 1 & 1 & 15 & 0 & 0 & 0 & 5 & 10 & 28 \\
\hline Vulnerable Road Users & 19 & 1 & 2 & 16 & 4 & 0 & 18 & 1 & 1 & 10 & 0 & 10 & 25 \\
\hline Traffic Simulation & 2 & 0 & 0 & 1 & 0 & 1 & 2 & 0 & 0 & 0 & 0 & 2 & 31 \\
\hline Overall & 156 & 10 & 21 & 127 & 29 & 12 & 156 & 10 & 3 & 34 & 41 & 95 & 28 \\
\hline
\end{tabular}

A

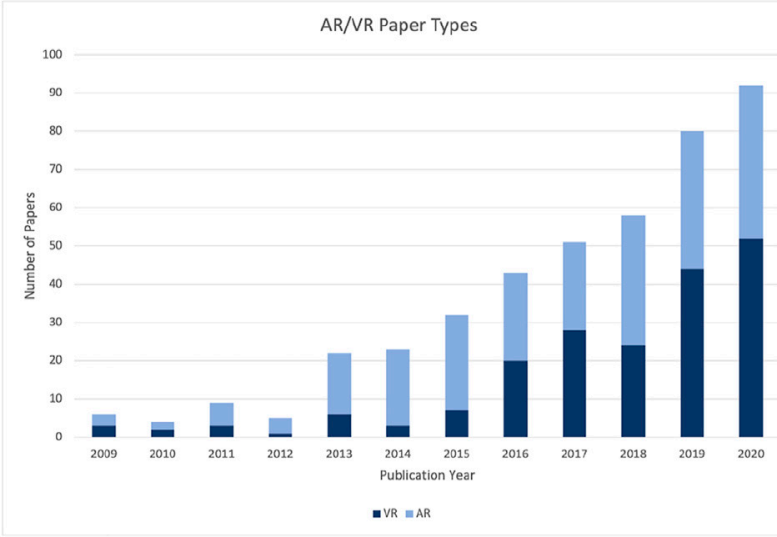

C

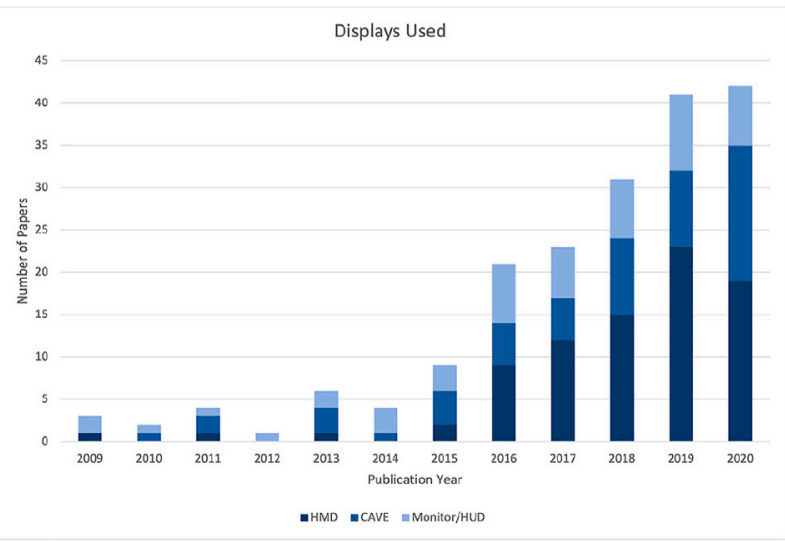

B

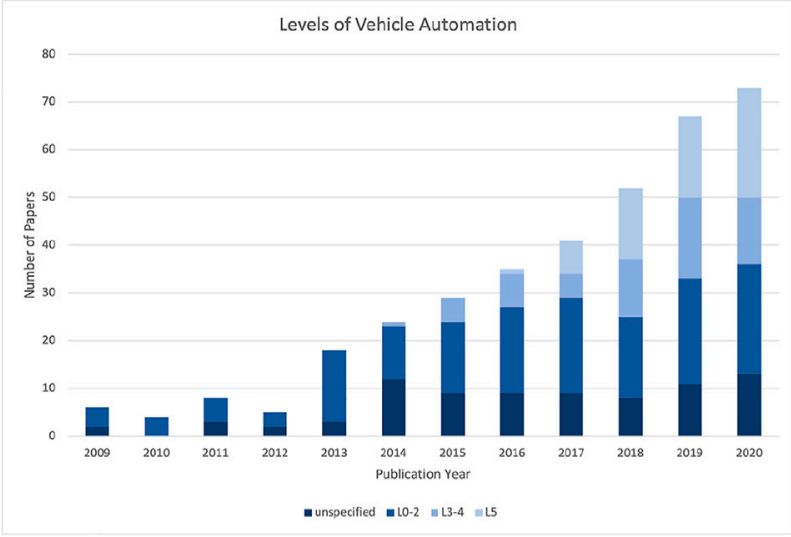

D

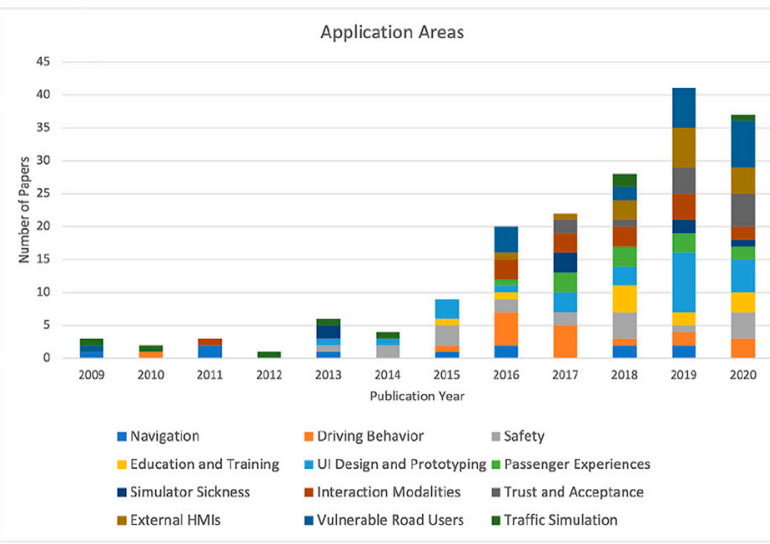

FIGURE 3 | High-level overview of the analyzed papers (A) AR/NR papers utilized in automotive research (B) Levels of vehicle automation (C) VR displays/devices used (D) Categorization of the papers into twelve application areas.

VR studies use a simplified version of traffic scenes, as well as their visual fidelity regarding pedestrians, and other road users, among others. While most papers provide screenshots of the VR environment, it is often unclear to what extent the VR scene resembles the real world, and real-world situations.
Consequently, this circumstance has a negative impact on the external validity of the user study findings, as there is no guarantee that they can be transferred to real environments, since VR driving simulators may not be realistic enough due to the limited field of view, or lack of motion feedback. As Yeo 
et al. (2020) found, mixed reality setups in real vehicles with motion feedback achieve the highest level of presence among automated driving simulators. However, conditions in realistic driving experiments are not as controlled or replicable as in a lab environment, and might differ in terms of surrounding traffic, weather conditions or vehicle speeds. Therefore, as computing and graphics power increase, we see the need to create "digital twins" of the real world (e.g., a section of a highway, a city borough) that feature content-rich real-world representations. Our review results also show that most studies conducted single sessions of interaction, and therefore only display snapshots of first time use. Even though usability has been shown to provide reliable information about user engagement within a single session experiment (Forster et al., 2019), user experience, and trust may require a longer time period to reach a robust behavior and attitude level (Lee and Moray, 1992). Longitudinal studies should therefore address these issues.

\subsection{Data Type}

We investigated the use of qualitative (subjective) and quantitative (objective) data types, and found that a total of 95 papers (56\%) collected both quantitative and qualitative data, 34 (20\%) papers only quantitative, and 41 (24\%) only qualitative. The predominantly used quantitative data involved driving performance (e.g., reaction time, braking distance), gaze and glance behavior, and physiological measurements, such as the heart rate variability and breathing metrics. Further, task performance (error/accuracy) was measured. As for subjective ratings, workload, user experience, simulator sickness, as well as custom self-ratings were reported. The concrete data types to collect often depend on the respective application area. For example, quantitative metrics such as braking and take-over performance are necessary for safety applications, while selfrated qualitative data such as acceptance and user experience metrics are more relevant for evaluating drivers'/passengers' trust and well-being. In VR setups, we identify the need to at least include one subjective measurement, which is the simulator sickness that determines the internal validity of the user study. Additionally, quantitative data such as eye or head tracking data can nowadays more easily be acquired using VR HMDs (such as the HTC Vive Pro Eye). Generally, we recommend using both quantitative and qualitative data types for conditionally automated driving studies (SAE level 3 and 4), as they should include take-over requests (i.e., measuring the take-over performance). Additionally, task performance and situation awareness can be quantitatively determined (effectiveness and efficiency according to ISO-9241 ISO, 2018). Encompassing user experience aspects, satisfaction as an attitudinal component (ISO, 2018) should be included using qualitative measurements such as self-rating questionnaires and interviews. For fully automated driving setups (SAE level 5), the focus of user studies will shift to in-vehicle experiences such as transforming the vehicle into a mobile office or entertainment platform (Schartmüller et al., 2018), and hence subjective ratings might be of higher importance. In general, we recommend to use method triangulation in user studies as described by Pettersson et al. (2018), including questionnaires (both standardized and custom), interviews, and activity logging. While the research by Pettersson et al. (2018) focus on user experience, we encourage to use method triangulation also for other application areas (see Section 4).

Further, the majority of the user studies was conducted in an indoor environment (156, or 92\%), while outdoor studies were made in 10 cases $(6 \%)$, and 3 studies reported a combination of both settings $(2 \%)$. These results seem plausible, as the use of virtual reality technology is currently still constrained by the lack of outdoor capabilities, such as power consumption and tracking devices. With the advances of wireless, battery-powered VR HMDs, however, coupled with the need to determine the external validity of VR indoor studies, we encourage researchers and practitioners to conduct user studies with this novel VR technology in outdoor settings.

\subsection{Participants}

The analysis of demographic data of the participants provided by the studies revealed that most studies were run with young participants from university populations. The mean age of participants utilized in the user studies was 29 years. While most user studies also included female participants (82\%, some studies did not explicitly report on gender), however, the ratio of female-male participants was quite low (34\% of total participants where a distinction between male and female subjects was specified). Regarding the recruitment source, we found that the majority of participants were recruited in universities (45\%), while public (10\%) and private company (3\%) recruitments were relatively rare. The remaining studies did not explicitly report the source of recruitment, which is an indication that many researchers may not be aware of reporting user study details, which limits their reproducibility and validity. The participant analysis shows that many VR user studies in the automotive domain are conducted with young male university students, as opposed to a more diverse and representative sample of the population. Our review provides a distinction between male and female participants only, as we did not identify any papers in our literature corpus that report additional genders. However, we encourage researchers and practitioners to include and report other genders as well to support inclusiveness. As most reported demographics were young, male students, an inherent bias is created in the study results, and thus, the external validity of the findings is limited. Additionally, the technical and technological affinity of these participants tends to be much higher than that of the general public, and its impact of their performance may be significant. We therefore urge experimenters to invest more effort in attracting female and other participants, as well as different age groups, cultural backgrounds, and levels of technological affinity to diminish the bias in the results, and foster their generalizability (Howard and Borenstein, 2018).

\subsection{Virtual Reality Devices and Fidelity}

Figure 3C gives an overview of devices used in the reported user studies, categorized into immersive HMDs and CAVEs, as well as non-to semi-immersive monitor/projection/HUD setups. While the usage of immersive displays has increased steadily since 2015, the usage of less immersive VR devices decreased. This shows an 
improved availability of advanced VR devices provided to researchers. Overall, approx. 82 papers (47\%) reported using HMDs, 54 (31\%) utilized CAVEs, and less immersive setups were used by 48 (27\%) of papers. This results in more than $100 \%$ as several studies utilized multiple VR setups. Overall, the vast majority of user studies was carried out by utilizing VR as a tool for prototyping different scenarios (94\%), while only $6 \%$ of the selected research papers would investigate the actual use of VR in the vehicle. A more detailed overview of utilized devices can be found in each application area in Section 4. While HMDs have a cost as well as portability benefit, CAVEs are not limited by a limited field of view, as projections are utilized to generate the VR environment. Over the last decade, VR technology became more affordable and easier to setup. Consequently, non-immersive VR setups, including monitors, should be avoided especially for continuous-depth applications such as simulated AR head-up or windshield displays. Additionally, the use and continuous improvement of open-source VR driving simulators (see Section 4.13) should be encouraged to consistently increase their fidelity, and democratization of scenarios and study setups.

\subsection{Levels of Vehicle Automation}

Figure $3 \mathbf{B}$ visualizes the total number of papers we investigated for the years 2009-2020, categorized by the researched level of vehicle automation, from manual (SAE L0) to fully automated driving (SAE L5). VR research on conditionally automated driving (SAE L3-4) was reported starting in 2015 with the emergence of VR HMDs of adequate quality for research. No vehicle automation was reported by 89 papers (50\%). Conditionally automated driving (SAE level 3 and 4) was researched by 28 papers (16\%), fully automated driving (SAE level 5) by $22 \%$, and research where the level of vehicle automation was not critical, and therefore not reported, was covered by $12 \%$ of the reviewed papers. Evidently, the utilization of AR/VR technology (Figure 3A) and research on higher levels of vehicle automation appear to go hand-in-hand, which seems to correspond to Gartner's hype cycle for emerging technologies (Gartner, Inc., 2014).

\subsection{Categorization}

We categorized the 176 into a total of twelve application areas which shows that there is a wide and open field for automotive researchers utilizing VR technology. Figure 3D gives an overview of the advancement of these application areas over time. Initial research with VR in the automotive domain concerned mostly navigation and driving behavior, as well as safety aspects. More recently, passenger experiences, vulnerable road users (such as pedestrians, and cyclists), as well as trust and acceptance of automated vehicles are the focus of research. Additionally, interaction concepts inside the vehicle and outside (external HMIs) are being investigated.

\subsection{Further Studies Information}

As shown in Table 1, most application areas had a similar mean number of authors for each paper, ranging between 3.00 (Simulator Sickness) and 3.88 (Interaction Modalities). However, papers in the Passenger Experiences area had the highest average number of authors (4.92), which highlights the interdisciplinary research field (automotive, human-computer interaction, and AR/VR visualization). Most papers were published at conferences venues, while for some application areas (Driving Behavior, Safety, and Trust and Acceptance) many studies were published in journals (approx. 50\%).

Overall, a total of 187 studies was reported in these 176 papers (Table 1), most of them (150, or 85\%) reported only one user study. The remaining papers reported two or more studies, including pilot studies. Regarding the median number of participants used, we found that Education and Training (52) and Safety (32) included the highest number of participants per study. Other application areas used between 20 and 30 participants per user study. Therefore, utilizing 20 to 30 participants in user studies seems to be a typical range in the VR automotive community.

In the following section, we review user studies and simulations in each of the twelve application areas separately. We further present overview tables, and discuss each application area by examining already conducted research, so that readers can get more detailed insights into user studies from that domain, as well as research gaps to find potential areas for future research.

\section{VIRTUAL REALITY APPLICATION AREAS}

\subsection{Vehicle Navigation and Routing}

A total of 11 studies were reported in the Vehicle Navigation and Routing application area. The majority of the studies investigated navigational aids for manual driving (see Table 3), although Sawitzky et al. (2019) presented an AR interface for fully automated vehicles. Interestingly, out of the 11 studies, six reported using CAVEs, three used HMDs, and two used some form of monitor/HUD display. This makes sense as navigational aids usually require a full view of the surrounding (virtual) environment, which CAVEs and HMDs offer. While eight studies were designed to be within-subjects, two studies were designed as mixed (within and between design). One pilot study did not specify the study design. On average, 20 participants joined a within-subject study, with approx. one third of the subjects being female. All studies were carried out in indoor locations. $75 \%$ of studies reported both objective (quantitative) and subjective (qualitative) data, while the rest focussed on qualitative results. Regarding objective data, error/accuracy and gaze metrics were reported prominently, while subjective feedback included self-ratings, NASA TLX, and perceived safety. Furthermore, all studies used VR as tool for conducting the experiment rather than as an end product.

\subsubsection{Discussion}

The conducted research in this application area is mostly considering manual and low levels of vehicle automation. With advancements of HMD and CAVE technology, navigation and routing tasks can more immersively be researched than monitors. Work needs to be done with regard to higher levels of vehicle automation, as motion sickness (covered in Section 4.7) needs to be investigated further, and 
TABLE 3 | Summary of user studies in Vehicle Navigation and Routing application area.

\begin{tabular}{|c|c|c|c|c|c|c|}
\hline References & Topic & $\begin{array}{l}\text { Displays } \\
\text { used }\end{array}$ & $\begin{array}{c}\text { Vehicle } \\
\text { automation }\end{array}$ & $\begin{array}{l}\text { Data } \\
\text { type }\end{array}$ & Dependent measures & $\begin{array}{l}\text { Participants } \\
\text { (female) }\end{array}$ \\
\hline Kim and Dey (2009) & $\begin{array}{l}\text { AR map on WSD, elderly } \\
\text { drivers }\end{array}$ & Monitor & LO-2 & $\mathrm{O}+\mathrm{S}$ & $\begin{array}{l}\text { Error/Accuracy, Rating, Gaze, Driving } \\
\text { time }\end{array}$ & $24(13)$ \\
\hline Sawitzky et al. (2019) & Traffic augmentation & HMD & L5 & S & TAM, TS, JB, Interview & $30(10)$ \\
\hline $\begin{array}{l}\text { Ying and Yuhui } \\
\text { (2011) }\end{array}$ & Chevron based navigation & Monitor & LO-2 & S & Rating & $39(9)$ \\
\hline Morley et al. (2016) & Smartphone use & HMD & LO-2 & $S$ & Rating, Interview & $22(14)$ \\
\hline Kraft et al. (2020a) & Cooperative driving & CAVE & LO-2 & $\mathrm{O}+\mathrm{S}$ & $\begin{array}{l}\text { Error/Accuracy, Rating, Gaze, } \\
\text { Perceived Safety }\end{array}$ & $24(13)$ \\
\hline Palinko et al. (2013) & AR navigation & CAVE & LO-2 & $O+S$ & $\begin{array}{l}\text { Error/Accuracy, Rating, NASA TLX, } \\
\text { Gaze }\end{array}$ & $10(\mathrm{NA})$ \\
\hline Bolton et al. (2015) & Landmark-based navigation & CAVE & LO-2 & $\mathrm{O}+\mathrm{S}$ & $\begin{array}{l}\text { Error/Accuracy, Rating, NASA TLX, } \\
\text { Gaze }\end{array}$ & $20(6)$ \\
\hline Jose et al. (2016) & $\begin{array}{l}\text { Comparison AR HUD, } \\
\text { HDD, HMD }\end{array}$ & HMD & LO-2 & $\mathrm{O}+\mathrm{S}$ & Error/Accuracy, Rating & $18(4)$ \\
\hline $\begin{array}{l}\text { Shahriar and Kun } \\
\text { (2018) }\end{array}$ & Camera-view AR & CAVE & LO-2 & $\mathrm{O}+\mathrm{S}$ & $\begin{array}{l}\text { Error/Accuracy, Rating, NASA TLX, } \\
\text { Gaze }\end{array}$ & $18(0)$ \\
\hline Topliss et al. (2018) & $\begin{array}{l}\text { Virtual lead vehicle as } \\
\text { navigational aid }\end{array}$ & CAVE & LO-2 & $\mathrm{O}+\mathrm{S}$ & Error/Accuracy, Rating, NASA TLX & $34(8)$ \\
\hline $\begin{array}{l}\text { Medenica et al. } \\
\text { (2011) }\end{array}$ & Comparing of navigation aids & CAVE & LO-2 & $\mathrm{O}+\mathrm{S}$ & $\begin{array}{l}\text { Error/Accuracy, Rating, NASA TLX, } \\
\text { Gaze }\end{array}$ & 18 (NA) \\
\hline
\end{tabular}

TABLE 4 | Summary of user studies in Driving Behavior application area.

\begin{tabular}{|c|c|c|c|c|c|c|}
\hline References & Topic & $\begin{array}{l}\text { Displays } \\
\text { used }\end{array}$ & $\begin{array}{c}\text { Vehicle } \\
\text { automation }\end{array}$ & $\begin{array}{l}\text { Data } \\
\text { type }\end{array}$ & Dependent measures & $\begin{array}{l}\text { Participants } \\
\text { (female) }\end{array}$ \\
\hline $\begin{array}{l}\text { Tateyama et al. } \\
(2010)\end{array}$ & Primary driving task & CAVE & LO-2 & $\mathrm{O}$ & Distance, Steering & $6(\mathrm{NA})$ \\
\hline Taheri et al. (2017b) & Primary driving task & HMD & LO-2 & $\mathrm{O}$ & $\begin{array}{l}\text { Steering, View Angle, Acceleration/ } \\
\text { Braking }\end{array}$ & 10 (NA) \\
\hline Blissing et al. (2019) & Driving behavior VR vs MR & HMD & LO-2 & $\mathrm{O}+\mathrm{S}$ & $\begin{array}{l}\text { Time, Distance, Acceleration/ } \\
\text { Braking, SSQ }\end{array}$ & $22(8)$ \\
\hline Chen et al. (2015) & Elderly drivers & HMD & LO-2 & $\mathrm{O}$ & Posture & $26(15)$ \\
\hline Blissing et al. (2016) & Visual latency & HMD & LO-2 & $\mathrm{O}+\mathrm{S}$ & $\begin{array}{l}\text { Time, Acceleration/Braking, Steering, } \\
\text { Rating }\end{array}$ & $24(6)$ \\
\hline Ali et al. (2016) & Gamification & $\mathrm{HMD}$ & LO-2 & $\mathrm{O}+\mathrm{S}$ & $\begin{array}{l}\text { Collisions, Speed, Error/Accuracy, } \\
\text { Rating }\end{array}$ & $13(7)$ \\
\hline Taheri et al. (2017a) & Driving characteristics & $\mathrm{HMD}$ & LO-2 & $\mathrm{O}$ & $\begin{array}{l}\text { Time, Distance, Acceleration/Braking, } \\
\text { Steering, Head position }\end{array}$ & $10(1)$ \\
\hline Walch et al. (2017) & Player perspective & $\begin{array}{l}\text { HMD, } \\
\text { Monitor }\end{array}$ & LO-2 & $S$ & SSQ, SAM, IEQ, PQ, Rating & $20(5)$ \\
\hline $\begin{array}{l}\text { Langlois and Soualmi } \\
\text { (2016) }\end{array}$ & AR take over & CAVE & L3-4 & $\mathrm{O}$ & $\begin{array}{l}\text { TOR Time, Distance, Acceleration/ } \\
\text { Braking }\end{array}$ & $26(0)$ \\
\hline Smith et al. (2016) & HUD vs HDD comparison & CAVE & LO-2 & $\mathrm{O}+\mathrm{S}$ & $\begin{array}{l}\text { Distance, Speed, Gaze, Error/Accuracy, } \\
\text { NASA TLX, Rating }\end{array}$ & $24(\mathrm{NA})$ \\
\hline Gerber et al. (2020) & NDRTs HUD vs Mobile & CAVE & L3-4 & $\mathrm{O}$ & Gaze, Time & $46(23)$ \\
\hline $\begin{array}{l}\text { Wang and Soffker } \\
\text { (2016) }\end{array}$ & Driving efficiency interface & Monitor & LO-2 & $\mathrm{O}+\mathrm{S}$ & Speed, Gaze, NASA TLX & $32(3)$ \\
\hline Uchida et al. (2017) & Driving performance evaluation & HMD & LO-2 & $\mathrm{O}$ & $\begin{array}{l}\text { Reaction time, Distance, Acceleration/ } \\
\text { Braking }\end{array}$ & $10(0)$ \\
\hline Yang et al. (2017) & $\begin{array}{l}\text { In-vehicle traffic lights for driving } \\
\text { performance }\end{array}$ & CAVE & LO-2 & $\mathrm{O}+\mathrm{S}$ & $\begin{array}{l}\text { Speed, Acceleration/Braking, Gaze, } \\
\text { Rating }\end{array}$ & $23(0)$ \\
\hline Wang et al. (2020) & AR driving assistance system & Monitor & LO-2 & $\mathrm{O}$ & Time, Distance, Fuel Consumption & NA \\
\hline Utsumi et al. (2018) & Emergency behavior & CAVE & L3-4 & $\mathrm{O}+\mathrm{S}$ & Error/Accuracy, Rating & $39(27)$ \\
\hline Kraft et al. (2020b) & Maneuver coordination & CAVE & LO-2 & $\mathrm{O}+\mathrm{S}$ & $\begin{array}{l}\text { Acceleration/Braking, Collisions, Gaze, } \\
\text { Rating }\end{array}$ & $68(32)$ \\
\hline Utsumi et al. (2019) & On-road virtual visual references & CAVE & LO-2 & $\mathrm{O}$ & Distance, Gaze & $34(27)$ \\
\hline
\end{tabular}

navigational aids (in combination with AR HUDs or WSDs) could prove helpful (e.g., Topliss et al., 2018). Further, the advent of AR technology can help to transition traditional, two- dimensional direction instructions towards world-relative three-dimensional instructions that blend into the outside environment (e.g., Ying and Yuhui 2011; Palinko et al., 2013; 
Bolton et al., 2015). Navigation is one area where AR technology could offer substantial advantages due to its ability to overlay virtual cues on top of the simulated world with continuous depth. This is becoming more and more relevant as AR displays become increasingly common in cars (e.g., head-up displays on the windshield) (Jose et al., 2016) and consumers begin to wear head-mounted displays outdoors. A novel future research direction for automated vehicles would be to integrate navigation tasks with passenger experiences. Navigation studies will also need to address the spatial capabilities of the vehicle and driver/passengers, the communication of depth cues, and spatial information display modalities (Gabbard et al., 2014). Further, future user studies should consider conducting outdoor navigation studies in a realistic test environment and the need to collect a range of qualitative and quantitative data.

\subsection{Driving Behavior}

A total of 18 studies were reported in the Driving Behavior application area. As expected, the majority of the studies investigated lower levels of vehicle automation (see Table 4), although three studies concentrated on conditionally automated driving (SAE L3-4). Out of the 18 studies, seven reported using CAVEs, seven used HMDs, and four used some form of monitor/ HUD setup. Only one study collected solely subjective data, as the majority of studies reported objective or mixed data. Among the quantitative data, distance, steering, acceleration/braking, and gaze metrics were reported highly, while subjective data included workload (NASA TLX), self-ratings, and SSQ (simulator sickness questionnaire). For the conditionally automated driving studies, take-over reaction times were additionally collected. While fifteen studies were designed to be within-subjects, two studies were designed as between and one as mixed. On average, 21 participants joined a within-subject study, with approx. one fourth of the subjects being female. Two of the studies were carried out in outdoor locations. Additionally, all studies used VR as tool for conducting the experiment rather than as an end product.

\subsubsection{Discussion}

Driving behavior is strongly associated with manual driving, focussing on the primary driving task. However, more recent concepts and studies show that non-driving related tasks are being investigated more extensively. As such, take-over requests (TORs), where the automated vehicle surrenders control to the human driver, will have to be researched more thoroughly. Keeping the driver "in the loop" is therefore one of the main tasks for driving behavior research. Gamification can be a great enabler in this area (Ali et al., 2016). A novel research approach would be to equip simulated automated vehicles with certain behaviors, such as "eco-driver" or "sporty driver". For example, novel interfaces could support the driver by giving constant feedback to the driver on their driving style (e.g., "ecofriendly"). In this regard, personalization concepts should be explored further, as user experience aspects will become more important in future vehicles (e.g., Riegler et al., 2020b; Lee et al., 2020). Furthermore, the inclusion of elderly drivers should receive more attention, and future in-vehicle interfaces should be tailored to this growing target group (Robertson and Vanlaar, 2008).

\subsection{Safety}

A total of 19 studies were reported in the Safety application area. The majority of the studies investigated safety aspects for manual driving (see Table 5), although six studies examined highly automated driving, and two studies evaluated mixed traffic situations, where both manual and automated vehicles share the road. Interestingly, only one study (Lindemann et al., 2018b) evaluated safety in the context of fully automated vehicles. Most studies regarded situation awareness and crash warnings, however, also smartphone usage and teleoperated driving were investigated. Out of the 19 studies, ten reported using CAVEs, four used HMDs, and five used some form of monitor/HUD display. While eleven studies were designed to be within-subjects, six studies were designed as between, and two as mixed. On average, 32 participants joined a within-subject study, with approx. one third of the subjects being female. All studies were carried out in indoor locations. About half of all studies reported both objective and subjective data, while the rest focussed on either quantitative or qualitative results. Regarding objective data, error/accuracy, collisions, distance, steering reversal rate (SRR), times such as reaction times, and time to collision (TTC), as well as gaze metrics were reported prominently. Physiological measures encompassed galvanic skin response (GSR), skin conductance response time (SCR), and heart rate (HR). Subjective feedback included user experience (AttrakDiff) and workload (NASA TLX), among custom questionnaires. Almost all reviewed studies all reported using VR as study setup. One study evaluated the use of VR HMDs for supporting teleoperations of shared automated vehicles, and one study used HMDs to alert drivers on safety-critical issues while using their smartphone while driving.

\subsubsection{Discussion}

Similar to the Navigation and Routing, and Driving Behavior application areas, the Safety category is dominated by research on manual driving. However, as mixed traffic situation will occur more prominently in the future, we believe that research interest will increase for highly automated vehicles, for example, when it comes to cooperative driving, and teleoperated driving. VR plays an important role in safety research, as AR awareness/attention cues can be simulated. As such, new opportunities will emerge encompassing both AR and VR in automotive safety research, including gamification concepts (Schroeter and Steinberger, 2016). As all reviewed papers in this category reported user studies being conducted indoors, opportunities emerge to research safety aspects in outdoor environments with VR HMDs, for example on test tracks (e.g., Frison et al., 2019). Additionally, several VR HMDs nowadays offer video/AR seethrough capabilities which could be researched for conditionallyand fully automated vehicles as modality to keep the driver in the loop. The review also shows that qualitative measurements are not as common as quantitative ones. When conducting further research in safety aspects, such as contact analogue warnings on a HUD, for example, we encourage researchers and practitioners to 
TABLE 5 | Summary of user studies in Safety application area.

\begin{tabular}{|c|c|c|c|c|c|c|}
\hline References & Topic & $\begin{array}{l}\text { Displays } \\
\text { used }\end{array}$ & $\begin{array}{c}\text { Vehicle } \\
\text { automation }\end{array}$ & $\begin{array}{l}\text { Data } \\
\text { type }\end{array}$ & Dependent measures & $\begin{array}{c}\text { Participants } \\
\text { (female) }\end{array}$ \\
\hline Morley et al. (2016) & $\begin{array}{l}\text { Smartphone use while } \\
\text { driving }\end{array}$ & HMD & LO-2 & S & Rating & $22(14)$ \\
\hline Rousset et al. (2015) & Distance perception & HMD & LO-5 & $\mathrm{O}$ & Error/Accuracy, Distance & $28(11)$ \\
\hline Brown et al. (2018) & Mixed traffic safety & HMD & LO-5 & $O+S$ & Collisions, GSR, Rating & $23(10)$ \\
\hline $\begin{array}{l}\text { Thomas and Charlton } \\
\text { (2020) }\end{array}$ & Inattentional blindness & CAVE & LO-2 & $\mathrm{O}$ & Error/Accuracy, Speed & $39(18)$ \\
\hline Kim et al. (2013) & Crash warning system & CAVE & LO-2 & $\mathrm{O}+\mathrm{S}$ & $\begin{array}{l}\text { Collisions, Reaction time, NASA TLX, } \\
\text { Rating }\end{array}$ & $16(8)$ \\
\hline Charissis (2014) & AR HUD warning & Monitor & LO-2 & $\mathrm{O}+\mathrm{S}$ & Collisions, Time & $20(10)$ \\
\hline $\begin{array}{l}\text { Häuslschmid et al. } \\
\text { (2015b) }\end{array}$ & AR WSD warning & Monitor & LO-2 & $\mathrm{O}+\mathrm{S}$ & $\begin{array}{l}\text { Distance, Time, Error/Accuracy, Gaze, } \\
\text { Rating }\end{array}$ & $18(2)$ \\
\hline Wulf et al. (2015) & Situation awareness & CAVE & L3-4 & $\mathrm{O}$ & Reaction time, Collisions, Gaze & 135 (NA) \\
\hline Hwang et al. (2016) & Risk perception & Monitor & LO-2 & $\mathrm{O}+\mathrm{S}$ & Response time, Rating & $31(1)$ \\
\hline Bout et al. (2017) & Teleoperation & HMD & L3-4 & $S$ & Rating & $12(2)$ \\
\hline $\begin{array}{l}\text { Schwarz and Fastenmeier } \\
(2017)\end{array}$ & AR warnings & CAVE & LO-2 & $\mathrm{O}+\mathrm{S}$ & $\begin{array}{l}\text { Acceleration/Braking, Gaze, AttrakDiff, } \\
\text { Rating }\end{array}$ & $88(18)$ \\
\hline Lindemann et al. (2018b) & Situation awareness & CAVE & L5 & $\mathrm{O}+\mathrm{S}$ & Error/Accuracy, Rating & $32(8)$ \\
\hline Wu et al. (2018b) & $\begin{array}{l}\text { Crash warning in fog } \\
\text { conditions }\end{array}$ & Monitor & LO-2 & $\mathrm{O}$ & $\begin{array}{l}\text { Collisions, Response time, } \\
\text { Acceleration/Braking }\end{array}$ & $54(\mathrm{NA})$ \\
\hline Lindemann et al. (2019) & $\begin{array}{l}\text { Short notice take-overs AR } \\
\text { warning }\end{array}$ & CAVE & L3-4 & $\mathrm{O}+\mathrm{S}$ & $\begin{array}{l}\text { Reaction time, Distance, Steering, } \\
\text { NASA TLX, Rating }\end{array}$ & $18(8)$ \\
\hline Yun and Yang (2020) & Multimodal warning HUD & CAVE & L3-4 & 0 & TOR time, Distance, SCR, HR & $41(16)$ \\
\hline Bram-Larbi et al. (2020) & Collision avoidance HUD & CAVE & LO-2 & S & Rating & $50(10)$ \\
\hline Calvi et al. (2020b) & AR for left turn maneuvers & CAVE & LO-2 & $\mathrm{O}$ & Error/Accuracy, Time & $46(13)$ \\
\hline Winkler et al. (2018) & Warning types & CAVE & LO-2 & $\mathrm{O}+\mathrm{S}$ & $\begin{array}{l}\text { Collisions, Acceleration/Braking, } \\
\text { Rating }\end{array}$ & $60(27)$ \\
\hline Lorenz et al. (2014) & AR take-over scenarios & Monitor & L3-4 & $\mathrm{O}$ & $\begin{array}{l}\text { TOR time, Acceleration/Braking, } \\
\text { Steering, Gaze }\end{array}$ & $46(10)$ \\
\hline Rusch et al. (2014) & AR cues for elderly drivers & Monitor & LO-2 & 0 & TTC, Time, Error/Accuracy & $64(29)$ \\
\hline
\end{tabular}

TABLE 6 | Summary of user studies in Driving Education and Training application area.

\begin{tabular}{|c|c|c|c|c|c|c|}
\hline References & Topic & $\begin{array}{l}\text { Displays } \\
\text { used }\end{array}$ & $\begin{array}{c}\text { Vehicle } \\
\text { automation }\end{array}$ & $\begin{array}{l}\text { Data } \\
\text { type }\end{array}$ & Dependent measures & $\begin{array}{c}\text { Participants } \\
\text { (female) }\end{array}$ \\
\hline Sportillo et al. (2018b) & $\begin{array}{l}\text { Comparison of educational } \\
\text { simulators }\end{array}$ & $\begin{array}{l}\text { HMD, } \\
\text { Monitor }\end{array}$ & L3-4 & $\mathrm{O}+\mathrm{S}$ & TOR time, Rating & 60 (NA) \\
\hline Sportillo et al. (2018a) & Comparison of training simulators & $\begin{array}{l}\text { HMD, } \\
\text { Monitor }\end{array}$ & L3-4 & $O+S$ & $\begin{array}{l}\text { TOR time, TTC, Distance, Gaze, } \\
\text { SSQ, Rating }\end{array}$ & $60(30)$ \\
\hline Sportillo et al. (2019) & Autonomous driving training & HMD & L3-4 & $\mathrm{O}+\mathrm{S}$ & TOR time, Rating & $60(31)$ \\
\hline $\begin{array}{l}\text { Pallamin and Bossard } \\
\text { (2016) }\end{array}$ & Presence \& realism & Monitor & LO-2 & $\mathrm{O}+\mathrm{S}$ & Error/Accuracy, IPQ & $36(10)$ \\
\hline Veronez et al. (2018) & Improve teaching/learning process & $\mathrm{HMD}$ & LO-2 & S & SUS & 52 (NA) \\
\hline $\begin{array}{l}\text { Likitweerawong and Palee } \\
\text { (2018) }\end{array}$ & Serious game as training & HMD & LO-2 & S & Rating & $100(25)$ \\
\hline Barić et al. (2020) & $360^{\circ}$ video-based training & HMD & LO-2 & S & Rating & $274(103)$ \\
\hline Mortimer et al. (2019) & Elderly drivers & HMD & LO-2 & $S$ & Rating & 37 (NA) \\
\hline Thevin et al. (2020) & People with visual impairments & HMD & LO-5 & $S$ & Rating & $13(5)$ \\
\hline Tudor et al. (2015) & People with spinal cord injury & CAVE & LO-2 & $S$ & Rating & $5(4)$ \\
\hline Sibi et al. (2020) & $\begin{array}{l}\text { Longitudinal training on driver } \\
\text { behavior and state }\end{array}$ & $\begin{array}{l}\text { Monitor, } \\
\text { CAVE }\end{array}$ & L3-4 & $\mathrm{O}$ & GSR, ECG, Drowsiness & $28(N A)$ \\
\hline
\end{tabular}

go beyond traditional measurements such as reaction, braking and take-over times, but also include user experience metrics using self-rating questionnaires (e.g., User Experience Questionnaire, AttrakDiff). Additionally, VR HMDs as tools for immersive teleoperations should be researched further, as initial research (e.g., Bout et al., 2017) shows promising results in spatial awareness for remote operators.

\subsection{Driving Education and Training}

We assessed 11 studies in the Driving Education and Training application area. Many of the studies investigated lower levels of vehicle automation (see Table 6), as educational driving simulators are evaluated for their application in driving schools or health centers. Nonetheless, four studies concentrated on highly automated driving. HMDs and 
TABLE 7 | Summary of user studies in UI Design and Prototyping area.

\begin{tabular}{|c|c|c|c|c|c|c|}
\hline References & Topic & $\begin{array}{l}\text { Displays } \\
\text { used }\end{array}$ & $\begin{array}{l}\text { Vehicle } \\
\text { automation }\end{array}$ & $\begin{array}{l}\text { Data } \\
\text { type }\end{array}$ & Dependent measures & $\begin{array}{c}\text { Participants } \\
\text { (female) }\end{array}$ \\
\hline Charissis et al. (2013) & WSD HMI design & Monitor & LO-2 & $\mathrm{O}$ & Collisions, Time & 20 (NA) \\
\hline Morra et al. (2019) & HMl design & HMD & L5 & $\mathrm{O}+\mathrm{S}$ & GSR, NASA TLX, VRUSE, SSQ, Rating & $38(13)$ \\
\hline Hock et al. (2016) & Visual feedback strategies & HMD & L3-4 & $\mathrm{O}+\mathrm{S}$ & Time, SSQ, Rating & $38(25)$ \\
\hline $\begin{array}{l}\text { Tesch and Dörner } \\
\text { (2020) }\end{array}$ & Vehicle interior inspection & $\mathrm{HMD}$ & L0-5 & $\mathrm{O}+\mathrm{S}$ & Time, NASA TLX, SSQ, Interview & $22(5)$ \\
\hline Gong et al. (2020) & Aid in manufacturing process & HMD & LO-5 & S & Rating, Interview & $14(\mathrm{NA})$ \\
\hline Merenda et al. (2019a) & Level of detail and visual fidelity & Monitor & LO-2 & $\mathrm{O}+\mathrm{S}$ & $\begin{array}{l}\text { Time, Acceleration/Braking, Gaze, } \\
\text { Rating }\end{array}$ & 24 (NA) \\
\hline Merenda et al. (2019b) & Level of detail and visual fidelity & Monitor & LO-2 & $\mathrm{O}+\mathrm{S}$ & $\begin{array}{l}\text { Speed, Steering, Gaze, NASA TLX, } \\
\text { Rating }\end{array}$ & $12(6)$ \\
\hline Paredes et al. (2018) & Prototyping VR Experiences & HMD & L5 & $\mathrm{O}+\mathrm{S}$ & HRV, BR, SSQ, TMS, Rating & $15(7)$ \\
\hline $\begin{array}{l}\text { Häuslschmid et al. } \\
(2015 a)\end{array}$ & WSD widgets \& personalization & Monitor & LO-2 & $O+S$ & $\begin{array}{l}\text { Error/Accuracy, Reaction Time, } \\
\text { AttrakDiff, Rating }\end{array}$ & $20(6)$ \\
\hline $\begin{array}{l}\text { Haeuslschmid et al. } \\
\text { (2017) }\end{array}$ & WSD text \& shape recognition & Monitor & LO-2 & $\mathrm{O}$ & Reaction time, Time & $46(11)$ \\
\hline $\begin{array}{l}\text { Häuslschmid et al. } \\
\text { (2019) }\end{array}$ & $\begin{array}{l}\text { Personalizing WSD content } \\
\text { presentation }\end{array}$ & Monitor & LO-2 & $\mathrm{O}+\mathrm{S}$ & Reaction time, Error/Accuracy, Rating & $35(13)$ \\
\hline Stockert et al. (2015) & Feedback design & Monitor & L3-4 & $\mathrm{O}+\mathrm{S}$ & $\begin{array}{l}\text { Reaction Time, Error/Accuracy, NASA } \\
\text { TLX, Rating }\end{array}$ & $21(7)$ \\
\hline Topliss et al. (2019) & Evaluating HUD locations & CAVE & LO-2 & $\mathrm{O}+\mathrm{S}$ & Speed, Distance, Steering, TTC, Rating & $26(9)$ \\
\hline Takada et al. (2019) & $\begin{array}{l}\text { Cognitive load for presenting } \\
\text { HUD information }\end{array}$ & Monitor & LO-2 & $\mathrm{O}$ & Reaction time, Error/Accuracy & $8(0)$ \\
\hline Feierle et al. (2019) & $\begin{array}{l}\text { Evaluating AR HUD in urban } \\
\text { driving }\end{array}$ & CAVE & L3-4 & $\mathrm{O}+\mathrm{S}$ & Gaze, NASA TLX, Rating & $30(10)$ \\
\hline $\begin{array}{l}\text { Amersfoorth et al. } \\
\text { (2019) }\end{array}$ & Translucency awareness cues & Monitor & LO-2 & $\mathrm{O}+\mathrm{S}$ & Reaction Time, Rating & $20(10)$ \\
\hline Li et al. (2020b) & HUD vs HDD NDRTs take-over & CAVE & L3-4 & $\mathrm{O}$ & Gaze & $46(23)$ \\
\hline Hensch et al. (2020) & Different display positions & CAVE & L3-4 & $\mathrm{O}+\mathrm{S}$ & Gaze, Error/Accuracy, Rating & $50(23)$ \\
\hline Topliss et al. (2020) & HUD time thresholds & CAVE & LO-2 & $\mathrm{O}+\mathrm{S}$ & TTC, Time, Gaze, Rating & $60(17)$ \\
\hline $\begin{array}{l}\text { Lindemann and Rigoll } \\
\text { (2017) }\end{array}$ & Diminished reality & CAVE & LO-2 & $\mathrm{O}+\mathrm{S}$ & Steering, View angle, Distance, Rating & $8(3)$ \\
\hline Ulahannan et al. (2020) & Information design preferences & CAVE & L3-4 & $S$ & Rating, Interview & $25(9)$ \\
\hline Smith et al. (2017) & $\begin{array}{l}\text { Eye scan patterns and HUD } \\
\text { perception }\end{array}$ & HUD & LO-2 & $\mathrm{O}+\mathrm{S}$ & $\begin{array}{l}\text { Time, TTC, Distance, Gaze, Error/ } \\
\text { Accuracy, NASA TLX, Rating }\end{array}$ & $16(8)$ \\
\hline $\begin{array}{l}\text { Lindemann et al. } \\
(2018 a)\end{array}$ & Explanatory WSD interface & CAVE & L5 & $S$ & Rating & $51(21)$ \\
\hline Bolder et al. (2018) & MR infotainment system & HMD & LO-2 & $\mathrm{O}+\mathrm{S}$ & $\begin{array}{l}\text { Time, Error/Accuracy, SUS, IPQ, SSQ, } \\
\text { Rating }\end{array}$ & $30(\mathrm{NA})$ \\
\hline Jakus et al. (2014) & HUD multimodal display & CAVE & LO-2 & $\mathrm{O}+\mathrm{S}$ & $\begin{array}{l}\text { Time, Collisions, NASA TLX, UEQ, } \\
\text { Rating, Interview }\end{array}$ & $30(9)$ \\
\hline Soro et al. (2014) & Augmented video & CAVE & LO-2 & $S$ & Interview & $11(\mathrm{NA})$ \\
\hline
\end{tabular}

monitors/HUDs were most commonly used, while a CAVE environment was only utilized in one study. All studies except one, which only gathered quantitative data, collected subjective data, and four studies (including all higher-level automation studies) additionally evaluated objective criteria, mostly TOR times. Physiological measurements included electrocardiogram signals (ECG) as well as galvanic skin response (GSR) data. As for qualitative data, self-ratings using custom questionnaires were used most extensively. Additionally, simulator sickness questionnaire (SSQ) and IGroup Presence Questionnaire (IPQ) were utilized. Six studies were designed as within-subject studies, and five as between-subject studies. The within-subject studies were conducted with 40 participants on average, with $43 \%$ of the subjects being female. With respect to the location, all studies took place in an indoor environment. Additionally, all studies used VR as tool for conducting the experiment rather than as an end product.

\subsubsection{Discussion}

As VR environments and scenarios can easily be tailored to their users' needs, VR systems are well suited for education purposes. For example, particular real-world situations that would be difficult to find, or replicate, can be modeled in VR, and even replicated as digital twins. Sportillo et al. (2018b) explored different educational simulators, and found that the acquisition of driving skills in conditionally automated vehicles was most highly rated for the VR solution, as compared to a user manual and a fixed-base simulator. Driving training in the context of mixed traffic might be especially useful for driving learners who have to consider cooperative driving situations with vehicles of different automation level, and driving simulators can help them achieve this paradigm shift (Sportillo et al., 2019). In addition, driving training can provide great benefits to people with visual and mental impairments to improve their orientation and mobility in road environments (Thevin et al., 2020). 
Therefore, we encourage automotive researchers to investigate health aspects as part of the education and training process in VR driving simulations. Moreover, we also see the need to conduct longitudinal studies in this domain, such as the one reported by Sibi et al. (2020). For example, future research could investigate longitudinal effects of future mixed traffic scenarios for novice drivers, and the effect on trust and acceptance of AVs, which shows the interdisciplinary nature of this application area.

\subsection{User Interface Design and Prototyping}

A total of 26 studies were reported in the UI Design and Prototyping application area. We found that HUD and WSD concepts were most prominently researched (see Table 7). Regarding vehicle automation, all manual, conditional, and fully automated driving scenarios were examined. Further, there was no clear preference towards utilized devices, as HMDs, CAVEs, and monitors/HUDs were employed. While 18 studies were designed to be within-subjects, four studies were designed as between, and four as mixed. On average, 24 participants joined a within-subject study, with $36 \%$ of the subjects being female. With the exception of two studies, all studies were carried out in indoor locations. The majority of all studies reported both objective and subjective data, while the rest focussed on quantitative results only. Regarding objective data, reaction times, error/accuracy, and gaze metrics were reported prominently, while subjective feedback included NASA TLX, SSQ, Toronto Mindfulness Scale (TMS), and selfratings. Additionally, most studies used VR as tool for conducting the experiment. However, three studies evaluated VR as end product such as a passenger wearing an HMD for content overlays synchronized with the vehicle's movements, and HMDs used as immersive instruments for vehicle interior and exterior design.

\subsubsection{Discussion}

Another application area of VR is the rapid UI design and prototyping process that can be achieved while being immersive and cost-effective Goedicke et al. (2018). For example, Morra et al. (2019), Riegler et al. (2020c), Charissis et al. (2013), and Gerber et al. (2020) use VR simulation to iteratively design AR HUDs or WSDs. Generally, vehicle interiors, surface quality, and shapes can be inspected in VR by using certain materials and shaders (Tesch and Dörner, 2020), and aid in the manufacturing process (Gong et al., 2020). Merenda et al. (2019a) and Merenda et al. (2019b) emphasize the level of detail and visual fidelity the virtual environment must have, as driving performance and gaze behavior are influenced by these parameters. Similarly, Paredes et al. (2018) suggest that VR experiences require a degree of sophistication even early on in the prototyping phase, which includes the use of high-fidelity content. We found that both qualitative and quantitative assessments were conducted for this application area, which we encourage to continue, as the performance of certain HMIs, but also their subjective ratings must be considered. In this regard, personalization should be researched further, as the emergence of more and more digital interfaces inside vehicles, and the concurrent discontinuation of hardware UIs (e.g., buttons, sliders) will allow novel tailored UIs to be crafted for any user.

\subsection{Passenger Experiences}

We investigated a total of 12 studies in the Passenger Experiences application area. Most of the studies investigated higher levels of vehicle automation (see Table 8), with special focus on fully automated driving reported by five studies. HMDs and monitors/ HUDs were commonly used, while a CAVE environment was only utilized in two studies. All studies collected qualitative data, and two studies additionally evaluated quantitative criteria, such as TOR times, and physiological data, including heart rate variability (HRV) and breathing rate (BR). Regarding subjective data, self-ratings using custom questionnaires were used most extensively, among simulator sickness questionnaires such as the SSQ and Engagement, Enjoyment, and Immersion (E2I) questionnaire. Nine studies were designed as within-subject studies, two as between-subject studies, and one as mixed-design. One study did not report the study design. The within-subject studies were conducted with 27 participants on average, with almost one third of the subjects being female. With respect to the location, two of the studies took place in an outdoor environment, the remaining studies were conducted in indoor lab environments. Nearly half of all papers utilized VR as an end product for investigating the use of HMDs for passengers, while the remaining papers employed VR to simulate AR content, for example.

\subsubsection{Discussion}

VR technology can further be employed to investigate passenger activities and experiences inside vehicles. Equivalent to windshield displays, side windows can also be used as displays utilizing corresponding technology to visualize content for side and back-seat passengers. In particular, Wang et al. (2016) investigated displaying entertainment and games on the side windows intended to keep younger passengers entertained on longer trips. Li J. et al. (2020) investigated back-seat commuters' preferences for activities in VR. Preferences indicate increased interest in productivity work, as being immersed in VR was perceived less distracting and more focussed. However, passengers should also be aware of physical borders to avoid bumping into the vehicle interior or other passengers, which should be researched further (proxemics, Li J. et al., 2020). VR HMDs can further enable passengers to "dive" into new (virtual) worlds in otherwise mundane drives (Paredes et al., 2018). To this end, Paredes et al. (2018) found that the proper match between VR content and the movement of the car can lead to significantly lower levels of motion sickness. Therefore, it is essential to match the speed of the VR content with that of the real vehicle. For highly automated driving, when drivers become passengers, this research will be especially valuable, as vehicles will be used as mobile entertainment or office platforms. We therefore encourage the automotive research community to investigate comfort, anxiety, entertainment, and work aspects, using virtual reality simulations for autonomous vehicles. As VR HMDs increase in portability, performance, and affordability, we believe that future in-vehicle experiences will include AR 
TABLE 8 | Summary of user studies in Passenger Experiences application area.

\begin{tabular}{|c|c|c|c|c|c|c|}
\hline References & Topic & $\begin{array}{l}\text { Displays } \\
\text { used }\end{array}$ & $\begin{array}{c}\text { Vehicle } \\
\text { automation }\end{array}$ & $\begin{array}{l}\text { Data } \\
\text { type }\end{array}$ & Dependent measures & $\begin{array}{l}\text { Participants } \\
\text { (female) }\end{array}$ \\
\hline Wang et al. (2016) & Entertainment and Games & CAVE & LO-5 & $S$ & Rating & NA \\
\hline Li et al. (2020a) & Backseat productivity work & HMD & L5 & $S$ & Rating & $11(1)$ \\
\hline Sakamura et al. (2018) & Boarding system for AV passengers & $\begin{array}{l}\text { HMD, } \\
\text { Monitor }\end{array}$ & L5 & $S$ & Rating & 20 (NA) \\
\hline Paredes et al. (2018) & Display virtual worlds & HMD & L5 & $\mathrm{O}+\mathrm{S}$ & HRV, BR, SSQ, Rating & $15(8)$ \\
\hline Koilias et al. (2019) & Anxiety & HMD & L5 & $S$ & Anxiety Modality Questionnaire & $75(31)$ \\
\hline $\begin{array}{l}\text { Ferrier-Barbut et al. } \\
(2018)\end{array}$ & Proxemics & HMD & L5 & $S$ & Rating & $19(4)$ \\
\hline Hock et al. (2017) & Entertainment & HMD & LO-5 & $S$ & SSQ, E2I, Rating & $23(5)$ \\
\hline Kodama et al. (2017) & Entertainment, car as motion platform & HMD & LO-5 & $S$ & Rating, Interview & $16(3)$ \\
\hline Wang et al. (2017) & Entertainment & Monitor & LO-5 & $S$ & Rating & 20 (NA) \\
\hline Arnedo et al. (2019) & Cross-vehicle multiplayer games & HMD & L3-4 & $\mathrm{O}+\mathrm{S}$ & $\begin{array}{l}\text { TOR time, PXI Questionnaire, } \\
\text { Interview }\end{array}$ & $12(3)$ \\
\hline Detjen et al. (2020) & $\begin{array}{l}\text { Reduce stress after passive accident } \\
\text { experiences }\end{array}$ & Monitor & L3-4 & S & Rating & $43(14)$ \\
\hline Wang et al. (2019b) & Rear passenger AR HUD & CAVE & LO-2 & $\mathrm{O}+\mathrm{S}$ & Collisions, Rating, TAM & $50(\mathrm{NA})$ \\
\hline
\end{tabular}

TABLE 9 | Summary of user studies in Simulator and Motion Sickness application area.

\begin{tabular}{|c|c|c|c|c|c|c|}
\hline References & Topic & $\begin{array}{l}\text { Displays } \\
\text { used }\end{array}$ & $\begin{array}{c}\text { Vehicle } \\
\text { Automation }\end{array}$ & $\begin{array}{l}\text { Data } \\
\text { type }\end{array}$ & Dependent measures & $\begin{array}{c}\text { Participants } \\
\text { (female) }\end{array}$ \\
\hline McGill et al. (2017) & Visual motion cues & HMD & L3-4 & S & SSQ, IPQ, Rating, Interview & $18(0)$ \\
\hline Sawabe et al. (2017) & Vection illusion & HMD & L5 & O & Vection & $9(0)$ \\
\hline Hanau and Popescu (2017) & Visual cues & HMD & L5 & S & MSAQ, Rating & $26(13)$ \\
\hline Williams et al. (2020) & $\begin{array}{l}\text { Visuo-haptic feedback } \\
\text { congruency }\end{array}$ & HMD & LO-2 & $\mathrm{O}+\mathrm{S}$ & Driving time, $P Q$ & $49(10)$ \\
\hline Lucas et al. (2020) & Seat vibrations & HMD & L5 & $\mathrm{O}+\mathrm{S}$ & Postural stability, SSQ, Rating & $30(6)$ \\
\hline $\begin{array}{l}\text { Jinjakam and Hamamoto } \\
\text { (2013) }\end{array}$ & $\begin{array}{l}\text { Parallax, position, height } \\
\text { difference }\end{array}$ & CAVE & LO-5 & $S$ & SSQ & $19(4)$ \\
\hline Karl et al. (2013) & $\begin{array}{l}\text { Simulator Sickness while } \\
\text { driving }\end{array}$ & HMD & LO-2 & $\mathrm{O}+\mathrm{S}$ & $\begin{array}{l}\text { Speed, Acceleration/ } \\
\text { Braking, SSQ }\end{array}$ & $44(15)$ \\
\hline Benz et al. (2019) & Comparison projector vs HMD & HMD, Monitor & LO-2 & S & $S S Q, P Q$ & $60(23)$ \\
\hline
\end{tabular}

content being displayed on the windshield and side windows, thereby superimposing digital objects onto the real world, as well as VR headsets providing immersive virtual content for rear-seat passenger. For the former, VR can be used to simulate augmentation concepts (e.g., Wang et al., 2017), while the latter used VR as end product.

\subsection{Simulator and Motion Sickness}

A total of 8 studies were reported in the Simulator and Motion Sickness application area. We found that research focussed on visual cues to tackle simulator sickness (see Table 9). Regarding vehicle automation, all manual, conditional, and fully automated driving scenarios were examined. Further, most user studies reported using HMDs, while two user studies used a CAVE or monitor/HUD setup, respectively. Similarly, an analysis of the collected data types shows that subjective criteria were evaluated most commonly, followed by mixed data types (3) and objectiveonly (1). Regarding objective data, driving times, error/accuracy, acceleration/braking, vection, and speed metrics were reported, while subjective feedback included SSQ, Presence Questionnaire (PQ), Motion Sickness Assessment Questionnaire (MSAQ), and self-ratings. All but one study reported using a within-subject design, as one study reported a between-subject design. On average, 29 participants joined a within-subject study, with only $23 \%$ of the subjects being female. Moreover, all studies were carried out in indoor locations, and most used the VR technology as tool rather than as an end product. In three studies, VR HMDs were utilized to investigate their applicability on displaying diminished or additional virtual content.

\subsubsection{Discussion}

When conducting user studies in VR in general, and on automated driving in particular, as active drivers become passive passengers, simulator and motion sickness must be considered. Simulator sickness is visually induced motion sickness (Rangelova and Andre, 2019). Motion sickness can occur when stationary users perform self-motion or when there are delays between head movements and the visual display presentation Hettinger and Riccio (1992). Known symptoms caused by motion sickness include nausea, disorientation, headache, and general discomfort, among others (Kennedy and Fowlkes, 1992). McGill and Brewster (2019) and McGill et al. (2017) explore the use of VR HMDs in vehicles worn by passengers. Motion sickness can occur 
TABLE 10 | Summary of user studies in Interaction Modalities application area.

\begin{tabular}{|c|c|c|c|c|c|c|}
\hline References & Topic & $\begin{array}{l}\text { Displays } \\
\text { used }\end{array}$ & $\begin{array}{l}\text { Vehicle } \\
\text { automation }\end{array}$ & $\begin{array}{l}\text { Data } \\
\text { type }\end{array}$ & Dependent measures & $\begin{array}{l}\text { Participants } \\
\text { (female) }\end{array}$ \\
\hline Manawadu et al. (2016) & Hand gestures & Monitor & L3-4 & $\mathrm{O}+\mathrm{S}$ & NASA TLX, Time, Rating & $20(7)$ \\
\hline Riegler et al. (2020a) & Head gaze & HMD & L3-4 & $\mathrm{O}+\mathrm{S}$ & NASA TLX, Time, Rating & $24(4)$ \\
\hline Riegler et al. (2020c) & Speech & HMD & L3-4 & $\mathrm{O}+\mathrm{S}$ & TOR Time, TAM, UEQ-S, SSQ, Interview & $23(11)$ \\
\hline Pan et al. (2017) & Brain signals & Monitor & LO-2 & $\mathrm{O}+\mathrm{S}$ & Error/Accuracy, Rating & 8 (NA) \\
\hline Halabi et al. (2017) & Body postures & HMD & LO-2 & 0 & Error/Accuracy & 20 (NA) \\
\hline $\begin{array}{l}\text { Moehring and Froehlich } \\
\text { (2011) }\end{array}$ & Grasping and Touching & HMD, CAVE & LO-2 & $\mathrm{O}+\mathrm{S}$ & Time, Rating, Interview & $12(1)$ \\
\hline Brand et al. (2016) & Pointing gestures at HUD & Monitor & LO-5 & $O+S$ & Pointing angle, Rating, Interview & $10(5)$ \\
\hline $\begin{array}{l}\text { Villalobos-Zúñiga et al. } \\
\text { (2016) }\end{array}$ & Keyboard & Monitor & LO-2 & $\mathrm{O}+\mathrm{S}$ & $\begin{array}{l}\text { Error/Accuracy, Time, Distance, Gaze, } \\
\text { NASA TLX }\end{array}$ & $16(6)$ \\
\hline Schartmüller et al. (2018) & Keyboard Input & CAVE & L3-4 & $\mathrm{O}+\mathrm{S}$ & $\begin{array}{l}\text { TOR time, Time, Steering, TTC, NASA } \\
\text { TLX, SSQ, Interview }\end{array}$ & $20(6)$ \\
\hline Lagoo et al. (2018) & Gestures & CAVE & LO-2 & $O+S$ & Error/Accuracy, Time, Rating & $20(7)$ \\
\hline Tippey et al. (2017) & $\begin{array}{l}\text { Hands-free interaction } \\
\text { with AR }\end{array}$ & Monitor & LO-2 & $\mathrm{O}+\mathrm{S}$ & $\begin{array}{l}\text { Steering Angle, Distance, Time, Error/ } \\
\text { Accuracy, Gaze, Rating }\end{array}$ & $24(9)$ \\
\hline Kun et al. (2019) & $\begin{array}{l}\text { Conversations } \\
\text { through AR }\end{array}$ & CAVE & LO-2 & $\mathrm{O}+\mathrm{S}$ & Distance, Gaze, Error/Accuracy, Rating & $10(2)$ \\
\hline Weidner and Broll (2019) & Finger gestures & Monitor & L3-4 & $O+S$ & Agreement Rate, SSQ, Rating & $23(11)$ \\
\hline Lagoo et al. (2019) & Gestures + HUD & CAVE & LO-2 & $O+S$ & Collisions, NASA TLX, Rating & $20(7)$ \\
\hline Wang et al. (2019a) & Gestures + HUD & Monitor & LO-5 & $S$ & Rating & $20(7)$ \\
\hline Prabhakar et al. (2019) & $\begin{array}{l}\text { Gaze and finger } \\
\text { controlled HUD }\end{array}$ & Monitor & LO-2 & $\mathrm{O}+\mathrm{S}$ & $\begin{array}{l}\text { Distance, Time, Steering, Gaze, SUS, } \\
\text { NASA TLX }\end{array}$ & $49(16)$ \\
\hline
\end{tabular}

through sensory mis-match which occurs when the eyes see that the digital content in VR does not conform to the vehicular movements registered by the vestibular system (Reason and Brand, 1975). Moreover, McGill et al. (2019) suggest that postural sway/head position changes should be kept to a minimum to reduce simulator sickness in case no positional tracking can be ensured by the simulator. Sawabe et al. (2017) use vection illusion to invoke pseudo-acceleration which leads to reduced acceleration stimulus, thereby helping passengers to counter simulator and motion sickness. A visual solution is proposed by Hanau and Popescu (2017) as they display visual cues of the acceleration to passengers, thus reducing the sensory conflict between the perceived acceleration and the absence of corresponding visuals. Williams et al. (2020) propose a multisensory setting, i.e., visuo-haptic feedback in VR driving simulation, and Lucas et al. (2020) explore seat vibrations and their effect on simulator sickness. Their findings show that feedback congruency and simulating road vibrations leads to more immersion for the driver, thereby creating a positive impact on simulator sickness. We see a wide range of proposed solutions to simulator/motion sickness being researched. It should be noted, that both physiological and subjective ratings (Simulator Sickness Questionnaire - SSQ, Presence Questionnaire - PQ) should be assessed and reported for user studies. Further, it is recommended to consider parameters such as study duration and visual fidelity in VR simulations, which influence the level of immersion and presence (Bowman and McMahan, 2007). Furthermore, as future in-vehicle experiences might include VR HMDs, we encourage researchers to explore their viability and applicability as end products for rear seat passengers, for example (McGill and Brewster, 2019). To this end, outdoor driving studies should be conducted, and congruency of visual and motion information, and lag effects should be researched.

\subsection{Interaction Modalities}

We examined a total of 16 studies in the Interaction Modalities application area. We found that research was conducted for both lower and higher levels of vehicle automation (see Table 10), and two studies reported details for all levels of vehicle automation. All VR devices (HMDs, CAVEs, monitor/HUDs) were utilized. Further, all but two studies collected both qualitative and quantitative data. Quantitative criteria included TOR times, error/accuracy, gaze, and steering metrics, among others. Qualitative data included NASA TLX, SSQ, self-ratings, and interviews, among others. 15 studies were designed as withinsubject studies, and one as between-subject study. The withinsubject studies were conducted with 19 participants on average, with about one third of the subjects being female. With respect to the location, all studies were conducted in indoor lab environments. Furthermore, all papers reported using VR as tool for conducting the study rather than as an end product.

\subsubsection{Discussion}

Future intelligent vehicles will need tailored modalities for interacting with their UIs. Instead of more traditional buttons, knobs, sliders and touch screens, novel forms of user interfaces (e.g., touch, speech, gestures, handwriting, and vision) Norman (2010) are researched further in the automotive domain. As Bottone and Johnsen (2016) state, one of the goals of VR driving simulation is to achieve high fidelity of interaction, maximizing the overall presence of the user in the simulation. This can be attained by providing real objects (e.g., pedals, steering wheel etc.) into the virtual environment. Manawadu 
TABLE 11 | Summary of user studies in Trust, Acceptance, and Ethics area

\begin{tabular}{|c|c|c|c|c|c|c|}
\hline References & Topic & $\begin{array}{l}\text { Displays } \\
\text { used }\end{array}$ & $\begin{array}{l}\text { Vehicle } \\
\text { automation }\end{array}$ & $\begin{array}{l}\text { Data } \\
\text { type }\end{array}$ & Dependent measures & $\begin{array}{l}\text { Participants } \\
\text { (female) }\end{array}$ \\
\hline $\begin{array}{l}\text { Shahrdar et al. } \\
\text { (2019) }\end{array}$ & $\begin{array}{l}\text { Evolution of trust between humans } \\
\text { and self-driving cars }\end{array}$ & HMD & L5 & $S$ & Rating & $50(8)$ \\
\hline Ha et al. (2020) & $\begin{array}{l}\text { Explanation types and perceived } \\
\text { risk on trust }\end{array}$ & HMD & L5 & $S$ & Rating & $51(18)$ \\
\hline $\begin{array}{l}\text { Djavadian et al. } \\
\text { (2020) }\end{array}$ & Acceptance of AVs & HMD & L5 & $S$ & Rating & $17(6)$ \\
\hline $\begin{array}{l}\text { Holländer et al. } \\
\text { (2019b) }\end{array}$ & $\begin{array}{l}\text { Communication between } \mathrm{AVs} \text { and } \\
\text { pedestrians }\end{array}$ & HMD & L5 & $\mathrm{O}+\mathrm{S}$ & Decision time, Rating & $18(11)$ \\
\hline Colley et al. (2020) & Recognizing pedestrians' intent & HMD & L5 & $\mathrm{O}+\mathrm{S}$ & $\begin{array}{l}\text { NASA TLX, Trust in Automation } \\
\text { Questionnaire, Rating, View Angle }\end{array}$ & $15(4)$ \\
\hline Samuel et al. (2020) & Ethical decision making & CAVE & LO-2 & $O+S$ & Rating, Gaze & $32(14)$ \\
\hline $\begin{array}{l}\text { Wintersberger et al. } \\
(2017)\end{array}$ & Traffic augmentation & CAVE & L5 & $\mathrm{O}+\mathrm{S}$ & HRV, TS, TAM, Interview & $26(11)$ \\
\hline $\begin{array}{l}\text { Wintersberger et al. } \\
\text { (2019) }\end{array}$ & Evaluating potential of $A R$ on trust & CAVE & L5 & S & UX Curve, TS, TAM, Interview & $18(6)$ \\
\hline $\begin{array}{l}\text { Häuslschmid et al. } \\
\text { (2017) }\end{array}$ & $\begin{array}{l}\text { Trust using avatar, WIM, and } \\
\text { indicators }\end{array}$ & Monitor & L5 & $S$ & UEQ, Rating, Interview & $30(12)$ \\
\hline Wu et al. (2020) & $\begin{array}{l}\text { AR interface - driver's attitude and } \\
\text { perception }\end{array}$ & Monitor & LO-2 & $S$ & Rating & $16(4)$ \\
\hline Uijong et al. (2019) & Ethical decision making & HMD & LO-2 & $\mathrm{O}+\mathrm{S}$ & HRV, Rating & $186(61)$ \\
\hline $\begin{array}{l}\text { Jayaraman et al. } \\
\text { (2018) }\end{array}$ & $\begin{array}{l}\text { Trust in AVs from a pedestrian } \\
\text { perspective }\end{array}$ & HMD & L5 & $\mathrm{O}+\mathrm{S}$ & Distance, Time, Speed, Rating & $30(N A)$ \\
\hline
\end{tabular}

et al. (2016) evaluate hand gestures as an alternative to the steering wheel in semi-automated vehicles. However, the majority of research on driver-vehicle interaction focuses on NDRTs instead of the primary driving task. For example, Riegler et al. (2020a) explore head gaze-based interactions with WSDs to interoperate with an infotainment system. Eye-tracking within VR has also been explored (Makedon et al., 2020). Riegler et al. (2020c) utilize speech-based interactions to perform a NDRT in semi-automated vehicles. Pan et al. (2017) used brain signals (i.e., electroencephalo-graph, or EEG) to let drivers navigate a vehicle in VR. Moreover, joysticks and similar hardware controllers already established to navigate $3 \mathrm{D}$ worlds were investigated (Chia and Balash, 2020). While we recognize that many interaction modalities are being researched separately, we encourage the utilization of sensor and interaction fusion, i.e., integrating multiple interaction modalities that are enabled according to the current context of being in a highly automated vehicle (e.g., work/leisure trip, entertainment/work, private/public transport, time of day). Additionally, different mobility concepts (e.g., shared public vehicles) should be evaluated in combination with accepted interaction types and their proxemics. In the areas of automotive context awareness, and personalization, in combination with mixed reality, we believe that research is still early and should be expanded.

\subsection{Trust, Acceptance, and Ethics}

A total of 12 studies were reported in the Trust, Acceptance, and Ethics application area. We found that fully automated vehicles were most prominently researched (see Table 11), however, also manual driving was researched in the area of ethical decision making. Further, HMDs and CAVE environments were employed in most studies, while monitor/HUD setups were utilized for two user studies. Eight studies were designed to be within-subjects, and four studies were designed as between studies. On average, 25 participants joined a within-subject study, with less than one third of the subjects being female. All studies were carried out in indoor locations. All studies reported subjective data, and half of the studies included objective data as well. Regarding objective data, HRV, decision times, and gaze metrics were reported prominently, while subjective feedback included Trust Scale (TS), Technology Acceptance Model (TAM), User Experience Questionnaire (UEQ), NASA TLX, and self-ratings. Moreover, all studies used VR as tool for conducting the experiment rather than as an end product.

\subsubsection{Discussion}

A number of research studies were carried out in VR environments to investigate trust in $\mathrm{AD}$ systems. Shahrdar et al. (2019) use a VR autonomous vehicle simulator to measure the evolution of trust between humans and selfdriving cars. To this end, they developed a real-time trust measurement to explore trust levels according to different driving scenarios. Similarly, $\mathrm{Ha}$ et al. (2020) examine the impact of explanation types and perceived risk on trust in AVs. Simple explanations, or feedback, such as descriptions of the vehicle's tasks, led to elevated trust in AVs, while too much explanation led to potential cognitive overload, and did not increase trust ( $\mathrm{Ha}$ et al., 2020). Djavadian et al. (2020) evaluate the acceptance of automated vehicles and found that especially heavy traffic situations lead to a higher acceptance as the vehicle would choose faster and less congested routes. Further, trust and acceptance should not only be evaluated from the driver's perspective, but also from an outsider's view. For example, Holländer et al. (2019b) look into communication 
TABLE 12 | Summary of user studies in External HMl application area.

\begin{tabular}{|c|c|c|c|c|c|c|}
\hline References & Topic & $\begin{array}{l}\text { Displays } \\
\text { used }\end{array}$ & $\begin{array}{c}\text { Vehicle } \\
\text { automation }\end{array}$ & $\begin{array}{l}\text { Data } \\
\text { type }\end{array}$ & Dependent measures & $\begin{array}{l}\text { Participants } \\
\text { (female) }\end{array}$ \\
\hline Singer et al. (2020) & AV parking intention and pedestrians & HMD & L5 & $\mathrm{O}+\mathrm{S}$ & Gaze, Time, Rating & $90(22)$ \\
\hline Asha et al. (2020) & $\begin{array}{l}\text { Vehicles as public displays, active route } \\
\text { navigation for pedestrians }\end{array}$ & HMD & LO-5 & S & Rating & $5(3)$ \\
\hline Böckle et al. (2017) & Shared AVs and pedestrians & HMD & L5 & S & Rating & $34(12)$ \\
\hline $\begin{array}{l}\text { Gruenefeld et al. } \\
\text { (2019) }\end{array}$ & Gestures between pedestrians and AVs & HMD & L5 & $\mathrm{O}+\mathrm{S}$ & $\begin{array}{l}\text { Error/Accuracy, NASA TLX, } \\
\text { Rating }\end{array}$ & $10(3)$ \\
\hline $\begin{array}{l}\text { Ackermans et al. } \\
\text { (2020) }\end{array}$ & Comparison eHMl - sensors & HMD & L5 & $\mathrm{O}+\mathrm{S}$ & $\begin{array}{l}\text { Error/Accuracy, Distance, } \\
\text { Rating, Interview }\end{array}$ & $64(31)$ \\
\hline Deb et al. (2018) & $\begin{array}{l}\text { Pedestrian suggestions for external features } \\
\text { on AVs }\end{array}$ & HMD & L5 & $\mathrm{O}+\mathrm{S}$ & Time, Error/Accuracy, Rating & $30(13)$ \\
\hline $\begin{array}{l}\text { Löcken et al. } \\
\text { (2019) }\end{array}$ & $\begin{array}{l}\text { Comparative analysis of eHMI interaction } \\
\text { concepts }\end{array}$ & HMD & L5 & $\mathrm{O}+\mathrm{S}$ & $\begin{array}{l}\text { Time, IPQ, UEQ-S, Rating, } \\
\text { Interview }\end{array}$ & $20(7)$ \\
\hline $\begin{array}{l}\text { Holländer et al. } \\
\text { (2019a) }\end{array}$ & $\begin{array}{l}\text { Influence of external car displays on } \\
\text { pedestrians' crossing behavior }\end{array}$ & HMD & L5 & $\mathrm{O}+\mathrm{S}$ & Time, Rating & $32(20)$ \\
\hline Rad et al. (2020) & $\begin{array}{l}\text { Pedestrians' road crossing behavior in front } \\
\text { of AVs }\end{array}$ & HMD & L5 & $\mathrm{O}+\mathrm{S}$ & ТTC, Rating, Interview & $60(20)$ \\
\hline Chang et al. (2017) & $\begin{array}{l}\text { Interface design for communication between } \\
\text { pedestrians and AVs }\end{array}$ & HMD & L5 & $\mathrm{O}+\mathrm{S}$ & $\begin{array}{l}\text { Error/Accuracy, Time, Rating, } \\
\text { Interview }\end{array}$ & $15(8)$ \\
\hline $\begin{array}{l}\text { Nguyen et al. } \\
\text { (2019) }\end{array}$ & $\begin{array}{l}\text { Projection-based communication between } \\
\text { pedestrians and AVs }\end{array}$ & HMD & L5 & S & Rating, Interview & $18(11)$ \\
\hline $\begin{array}{l}\text { Hudson et al. } \\
\text { (2018) }\end{array}$ & Pedestrian perception of AVs with eHMls & HMD & L5 & $S$ & SSQ, Rating & $16(7)$ \\
\hline Stadler et al. (2019) & $\begin{array}{l}\text { Communication between pedestrians } \\
\text { and } \mathrm{AVs}\end{array}$ & HMD & L5 & $\mathrm{O}+\mathrm{S}$ & Error/Accuracy, Time, Rating & $18(\mathrm{NA})$ \\
\hline Clercq et al. (2019) & Pedestrian crossing decisions & HMD & L5 & S & Rating, Interview & $28(7)$ \\
\hline $\begin{array}{l}\text { Otherson et al. } \\
\text { (2018) }\end{array}$ & $\begin{array}{l}\text { eHMI perspectives from older and younger } \\
\text { persons }\end{array}$ & $\mathrm{HMD}$ & L5 & $\mathrm{O}+\mathrm{S}$ & Time, UEQ, Rating, Interview & $43(19)$ \\
\hline
\end{tabular}

TABLE 13 | Summary of user studies in Vulnerable Road Users application area.

\begin{tabular}{|c|c|c|c|c|c|c|}
\hline References & Topic & $\begin{array}{l}\text { Displays } \\
\text { used }\end{array}$ & $\begin{array}{l}\text { Vehicle } \\
\text { automation }\end{array}$ & $\begin{array}{l}\text { Data } \\
\text { type }\end{array}$ & Dependent measures & $\begin{array}{l}\text { Participants } \\
\text { (female) }\end{array}$ \\
\hline Mallaro et al. (2017) & HMD vs CAVE & HMD, CAVE & LO-5 & $\mathrm{O}$ & Time & $32(16)$ \\
\hline $\begin{array}{l}\text { Feldstein and Dyszak } \\
\text { (2020) }\end{array}$ & Real vs VR comparison & HMD & LO-2 & $\mathrm{O}$ & TाC & $30(11)$ \\
\hline Wu et al. (2009) & Roundabout crossing behavior & HMD & LO-2 & $\mathrm{O}+\mathrm{S}$ & Error/Accuracy, Time & $12(6)$ \\
\hline Doric et al. (2016) & Crossing behavior & HMD & LO-5 & $\mathrm{O}+\mathrm{S}$ & Velocity, Rating & $6(2)$ \\
\hline Wu et al. (2018a) & $\begin{array}{l}\text { Roundabout crossing behavior, } \\
\text { Focus on sound }\end{array}$ & $\mathrm{HMD}$ & LO-5 & $\mathrm{O}$ & Time & $16(8)$ \\
\hline Holdgrun et al. (2018) & Crossing behavior & HMD & LO-5 & $\mathrm{O}$ & Collisions, Body Posture & 23 (NA) \\
\hline Perez et al. (2019) & Crossing behavior using AR & HMD & LO-5 & $\mathrm{O}$ & Time, Distance & 5 (NA) \\
\hline Velasco et al. (2019) & Crossing behavior & $\mathrm{HMD}$ & L5 & $\mathrm{O}+\mathrm{S}$ & $\begin{array}{l}\text { Distance, Presence Questionnaire, } \\
\text { Rating }\end{array}$ & $55(23)$ \\
\hline Ye et al. (2020) & $\begin{array}{l}\text { Crossing behavior, unfamiliar } \\
\text { driving rules }\end{array}$ & CAVE & LO-5 & $\mathrm{O}+\mathrm{S}$ & Error/Accuracy, TTC, Gaze, SSQ, Rating & $102(59)$ \\
\hline $\begin{array}{l}\text { Schrom-Feiertag et al. } \\
\text { (2019) }\end{array}$ & Speed perception & HMD & LO-5 & $\mathrm{O}+\mathrm{S}$ & Speed & $20(10)$ \\
\hline Pugliese et al. (2020) & Speed perception & CAVE & L5 & $\mathrm{O}$ & Time, Speed & $81(48)$ \\
\hline Colley et al. (2020) & Pedestrian intent recognition & HMD & L5 & $\mathrm{O}+\mathrm{S}$ & $\begin{array}{l}\text { View angle, NASA TLX, SUS, Trust in } \\
\text { Automation Questionnaire }\end{array}$ & $15(4)$ \\
\hline Bozkir et al. (2019) & Driver attention & HMD & LO-2 & $\mathrm{O}$ & Distance, Acceleration/Braking, Gaze & $16(4)$ \\
\hline Phan et al. (2016) & Driver awareness & Monitor & LO-2 & $\mathrm{O}+\mathrm{S}$ & $\begin{array}{l}\text { Time, Distance, Acceleration/Braking, } \\
\text { Rating }\end{array}$ & $27(4)$ \\
\hline Merenda et al. (2017) & Driver awareness & CAVE & LO-2 & $\mathrm{O}+\mathrm{S}$ & $\begin{array}{l}\text { Time, Distance, Acceleration/Braking, } \\
\text { Gaze, Rating }\end{array}$ & $24(9)$ \\
\hline Frémont et al. (2019) & Driver awareness & Monitor & LO-2 & $\mathrm{O}+\mathrm{S}$ & Time, Acceleration/Braking, Rating & $23(4)$ \\
\hline Calvi et al. (2020a) & Driver awareness & CAVE & LO-2 & $\mathrm{O}+\mathrm{S}$ & $\begin{array}{l}\text { TTC, Distance, Acceleration/Braking, } \\
\text { Rating }\end{array}$ & $46(13)$ \\
\hline Karatas et al. (2020) & Driver awareness & Monitor & LO-2 & $\mathrm{O}+\mathrm{S}$ & Speed, Gaze, Rating & $36(10)$ \\
\hline Pichen et al. (2020) & Driver awareness on cyclists & CAVE & LO-2 & $\mathrm{O}$ & Distance & $30(11)$ \\
\hline Maruhn et al. (2020) & Pedestrian behavior in AR & HMD & L5 & $\mathrm{O}+\mathrm{S}$ & Time, Perceived Safety, Rating & $30(15)$ \\
\hline
\end{tabular}


concepts between AVs and pedestrians and their impact on trust by using VR simulations. In particular, they look at crossing scenarios and how AVs can let pedestrians know when it is safe to cross the street. Additionally, Colley et al. (2020) explore trust in AVs by detecting and recognizing pedestrians' intent in order to calibrate driver's trust. As challenges in this application area, we see the need to measure trust over longer periods of time, and the possibility of decreasing and subsequently re-building trust in automated vehicles. Context awareness that is influenced by the driver's physiological metrics (e.g., stress), could be used to adapt (i.e., increase or decrease) system feedback and information display.

\subsection{External Human-Machine Interfaces}

We examined a total of 15 studies in the External HMI application area. We found that research was conducted almost exclusively for fully automated vehicles (see Table 12). All studies utilized HMDs for their HMI concepts. Further, all studies collected qualitative data. Additionally, quantitative data was reported by eight studies. Quantitative criteria included time measurements, error/accuracy, and gaze metrics, among others. Qualitative data included NASA TLX, User Experience Questionnaire (UEQ-S), self-ratings, and interviews, among others. Most studies were designed as within-subject studies, and were conducted with 32 participants on average, with $43 \%$ of the subjects being female. With respect to the location, all studies were conducted in indoor environments. In addition, all these studies utilized VR as tool for conducting the research rather than using it as an end product.

\subsubsection{Discussion}

Several studies in automotive research exist that focus on external car bodies as a design space. Since the technical implementation for real vehicles is cumbersome and costly, the majority of research utilizes VR to explore external HMI (eHMI) concepts. External automotive interfaces provide services to different road users, such as pedestrians, passengers, and drivers of other vehicles. In mixed traffic situations, where automated and non-automated vehicles share the road, eHMIs can provide additional information, such as the driving state and intent, to other road users. In a VR simulator study, Singer et al. (2020) explore a communication interface between the automated vehicle intending to park, and pedestrians. The user study results reveal that additional signals achieve a better perception of the vehicle's intention, and improve perceived safety. As such, external HMIs provide a novel design space for pedestrians. Asha et al. (2020) investigate various eHMI concepts, such as public displays, warning systems, and malfunction alerts. Additionally, eHMIs can be used as ambient displays by blending into the environment to reduce visual pollution in the cityscape (Asha et al., 2020). However, such eHMIs are not limited to visualization only (e.g., Chang et al., 2017; Stadler et al., 2019), indeed, they can be used for interaction purposes, such as active route navigation for pedestrians using gesture interaction (Gruenefeld et al., 2019). We encourage researchers to investigate eHMIs not only for pedestrians, but also for cyclists to enable safe interactions, and foster social connections between automated vehicles and vulnerable road users, for example by defining a common understanding, or guidelines, between them Kaß et al. (2020). Additionally, our review shows that most eHMI research is focused on SAE L5 fully automated vehicles, however, conditionally automated vehicles need to communicate with pedestrians as well, as the driver might not be fully attentive to the road environment, yet still being present in the vehicle. For example, SAE L3 and L4 vehicles could monitor the driver's attentiveness towards the outside environment, and accordingly visualize the eHMI to pedestrians.

\subsection{Vulnerable Road Users}

A total of 20 studies were reported in the Vulnerable Road Users (VRU) application area. We found that pedestrian behavior, especially crossing behavior with automated vehicles, was most prominently researched. In addition, the driver perspective regarding pedestrian safety was also extensively researched. As such, the research in this application area was applied to all levels of vehicle automation (see Table 13). Further, HMDs and CAVE environments were employed in most studies, while monitor/ HUD setups were utilized for three user studies. Sixteen studies were designed to be within-subjects, and four studies were designed as between studies. On average, 32 participants joined a within-subject study, with approx. $43 \%$ of the subjects being female. All studies were carried out in indoor locations, one study included an outdoor setup in addition, and one study was solely outdoor-based. All studies reported objective data, and about half of the studies included subjective ratings as well. Regarding objective data, time and distance measurements, speed, acceleration/braking, and gaze metrics were reported prominently, while subjective feedback included NASA TLX, System Usability Scale (SUS), Trust in Automation Questionnaire, and self-ratings. Furthermore, all studies made use of VR as tool, rather than as an end product.

\subsubsection{Discussion}

Vulnerable road users, including pedestrians and cyclists, are being researched from two perspectives: 1 ) the driver perspective that has the responsibility to be aware of the surroundings in manual driving (e.g., Bozkir et al., 2019; Frémont et al., 2019), and 2) the VRU perspective that needs to understand the driver/ vehicle intentions (e.g., Doric et al., 2016; Velasco et al., 2019). As such, speed perceptions and crossing behaviors have to be thoroughly evaluated. With the emergence of highly automated vehicles, we recognize that drivers might be engaged in NDRTs, such as entertainment or work activities (Pfleging et al., 2016). The communication of situation awareness even with higher levels of vehicle automation must therefore be researched more, including feedback strategies and visual, auditory, or multimodal transitions from the NDRT to the attention-required task. Therefore, take-overs need to be carefully explored, and we encourage researchers to investigate both quantitative and qualitative measures. Particularly, augmented reality head-up and windshield displays visualizing hazard cues to the driver could prove beneficial for the safety of VRUs (e.g., Phan et al., 2016). The possibility to simulate AR cues in VR environments enables researchers with new ways to explore VRU scenarios in safe and controlled study settings. 


\subsection{Traffic Simulation}

In this application area, virtual reality is used to generate and analyze traffic scenarios (e.g., traffic jams, throughput at intersections, among others). Throughout the analysis we identified 11 out of 176 papers which are focusing on traffic simulations and gave them more attention. We omitted an overview table, since only two of these papers included user study findings. Ayres and Mehmood (2009) developed an opensource traffic simulator capable of generating many permutations and adjustments of parameters to simulate real life highway situations. The advantages of using VR are 1) to visualize the virtual road traffic environment in real time in an immersive manner from different perspectives, and 2) displaying the data generated from the virtual sensors and creating graphical renderings, such as graphs and charts. In the context of highway scenarios, $\mathrm{Xu}$ et al. (2020) investigate merging conflicts visualized in the VR environment rather than in traditional microscopic simulations for a more immersive analysis. Traffic simulations focussing on city traffic are conducted by Grasso et al. (2010), who evaluate commute times as well as eco-friendliness of various traffic simulations. Luo et al. (2012) developed a VR traffic simulation with emphasis on weather effects, such as cloudy and icy conditions, as numerous traffic accidents can be traced back to harsh climate or complicated terrain conditions. Yu et al. (2014) further consider wind effects in their microscopic VR traffic simulator by calculating aerodynamic parameters in order to optimize intelligent traffic throughput. Additionally, Yu et al. (2013) investigate overtaking behaviors in their VR traffic simulator by modeling lane changing behavior. In order to verify the simulation correctness and effectiveness of their model, Yu et al. (2013) evaluated complex traffic maneuvers. A user study on lane-change behavior conducted by Zimmermann et al. (2018) showed that game-theoretic approaches have the potential to power local cooperation between traffic participants using social interaction concepts. Mixing the virtual and real world, using so-called digital twins, is accomplished by Szalai et al. (2020) in the form of testing a real vehicle in a virtual environment. While the utilization of traffic simulation provides a large number of possible scenarios, a test vehicle with its full control algorithm can be evaluated in various traffic situations. With the mixed reality or digital twin approach, Szalai et al. (2020) state that simulation environments can greatly support the automated vehicle development process, and facilitate validation procedures for such systems.

\subsubsection{Discussion}

We see great potential for digital twin approaches in mixed traffic simulations and user studies, where study participants can experience close-to-reality scenarios. Potential application areas are driving education and training, as fuel consumption, energy efficiency etc. can be calculated in real time. We further see the need to develop heuristics in order to evaluate scenarios according to a set of parameters, and allow for reproducibility and validity evaluation of simulations. To this end, we believe it is essential to share datasets and make evaluation results available open-source to foster synergies between researchers.

\subsection{Virtual Reality Driving Simulators}

Overall, a rise in VR user studies for a growing number of use cases can be identified. Accordingly, the role, importance, and responsibility of VR driving simulators is increasing as well. While early VR driving simulators were monitor-based (e.g., Weinberg et al., 2011; Yang et al., 2016), more recent research is conducted with more immersive solutions, such as HMDs and CAVEs. For example, Ihemedu-Steinke et al. (2017) and Grasso et al. (2020) provide details into the conceptionalization, architectural design, modeling, and implementation phases of creating a VR driving simulator using HMDs. More details on a CAVE infrastructure for VR driving simulation with focus on investigating AR HUDs are presented by Gabbard et al. (2019). While most VR driving simulators are placed in indoor lab environments, for example, Ghiurãu et al. (2020) investigate see-through $\mathrm{AR}$ concepts in real vehicle while the driver is wearing a VR HMD. A recent comparative study between six different autonomous driving simulation platforms with varying levels of visual and motion fidelity conducted by Yeo et al. (2020) shows that MR and VR HMDs surpass simple monitor setups for driving simulators, even more so, when applied in a real vehicle. Additional research is being conducted on the use of hardware controllers (Sportillo et al., 2017), and natural interaction metaphors (Moehring and Froehlich, 2011) within VR driving simulator setups.

Moving forward, we recommend readers to consider the use of Open Source VR driving simulators, because of the research community support, reproducibility, and validity for user studies. Several solutions exist, which we outline in the following:

- AutoWSD (Riegler et al., 2019a; Riegler et al., 2019a): focus on rapid prototyping of HCI concepts (interaction modalities, windshield displays) in automated driving (SAE L3-5) using customizable scenarios.

- CARRS-Q IVAD Simulator (Schroeter and Gerber, 2018; Gerber et al., 2019): focus on rapid prototyping of HCI concepts for SAE L3-5 AVs based on pre-recorded $180 / 360^{\circ}$ videos.

- VR-OOM (Goedicke et al., 2018): focus on evaluating HCI concepts in VR in a real vehicle.

- OpenROUTS3D (Neumeier et al., 2019) focus on teleoperated driving to help overcome the problems of automated driving.

- AirSim (Shah et al., 2017): focus on physically and visually realistic simulations for autonomous vehicles and drones.

- GENIVI (GENIVI Vehicle Simulator, 2016): focus on development and testing of in-vehicle infotainment systems.

\section{CONCLUSION}

\subsection{Overall Summary}

In this paper, we reported on the status quo of VR applications utilized in human factors research in driving automation, from 2009 to 2020 . We reviewed papers from a wide range of journals and conferences, which included 176 papers. We followed a structured approach to give an overview of the research 
domain by selecting relevant papers, and reviewing them in a standardized manner. In the continuously growing number of papers over the years 2009-2020, our exploration shows that as increasing levels of vehicle automation are researched, the usage of VR applications is utilized accordingly. There is a good portion of research in different aspects of VR utilization in driving automation indicating that researchers in the community work on the issue of developing and improving human-machine interfaces for automated vehicles and entities affected by them, such as drivers, passengers, pedestrians, and other road users. When researchers and practitioners plan to engage in research and development of automated driving, the present work provides them with an overview of the current landscape. Thus, one can derive information about main research areas and emerging trends that have not been studied extensively so far.

\subsection{Findings and Suggestions}

Through our literature survey, we identified gaps in the existing research, and condensed these findings into what we see as the current high-level challenges in this domain. This review suggests opportunities for improved user study designs, more use of field/ outdoor studies, and an increased number of evaluation methods. We found that user studies are conducted with predominantly young, male, and technologically educated participants, which indicates the need for more inclusive and diverse selection of participants. We also remark that within-subject designs are commonly used in VR, and as such need fewer participants to reach adequate statistical significance, as compared to betweensubject designs. The median number of participants (at least 20 participants) seems to be adequate for all application areas. Overall, formal, lab-based experiments dominate VR studies, however, automotive VR researchers and practitioners are encouraged to seek to perform more studies in outdoor, realworld usage scenarios, as the social and environmental effects of outdoor locations may vary from indoor sites. Hence, results received from indoor studies cannot be immediately applied to outdoor locations. Finally, as VR technology becomes consumergrade and more wide-spread across households, their usage not only as a tool, but as an end product should be further explored.

This literature review aims to provide researchers and practitioners with recommendations about methodological approaches (i.e., collection methods and specific parameters) that can be followed when conducting VR user studies for automated driving research. To conclude, we list a set of suggestions to be taken into consideration in future study setups utilizing virtual reality technology in the automotive domain:

- User study design and reporting. As this literature review shows, many user studies are carried out with an overwhelming majority of young, male participants from universities' populations. Additionally, we found that reporting on female and other genders is often omitted which indicates a lack of inclusiveness in this research area. Moreover, any user study without details on participant population, demographics, study design, or experimental tasks, is difficult to replicate, and their results can hardly be generalized. Finally, we also propose to specify the paper keywords according to the study setup, i.e., if a virtual reality driving simulator was utilized, we encourage to add this information in the keywords or abstract section. This indicates a general requirement to raise the reporting quality of user studies and to instruct researchers in the field on how to conduct good VR user studies.

- Heuristics, benchmarking, and datasets. The establishment of common criteria to test HMI designs (e.g., structural user interface metrics) should be pursued, in order to automate design evaluations, and need less reliance on lengthy user studies. Further, datasets should be shared to allow comparisons between studies, and improve upon these criteria.

- Use of standardized questionnaires and open-source VR driving simulators. In order to foster the comparison of experiment results, we suggest using established measurements and standardized questionnaires in terms of used parameters, calculation and reporting. Additionally, aiming at increased replicability of user studies, we recommend utilizing and contributing to open-source VR driving simulators.

- Additional focus on user experience. Considering particular research questions, we recommend exploring constructs like personalization and well-being, besides usual research topics such as safety, trust \& acceptance, and situation awareness. Furthermore, user-centered design of vehicular functions, and focus on user experience, might help to advance the acceptance of automated vehicles in the public (Frison et al., 2017).

- Fidelity and realism. As technological aspects of VR devices such as field of view, resolution, and processing power improve, we suggest to visually enhance the virtual environments, and aim to create digital twins of parts of the real world to foster higher levels of realism.

- Measuring simulator sickness. When conducting experiments in VR driving simulators, or with VR devices in general, we suggest assessing study participants' simulator sickness before and after the experiment using standardized questionnaires (e.g., SSQ). While we found that many researchers utilize such questionnaires at the end of the experiment, we propose to use them also prior to the VR simulation in order to analyze its impact on participants' nausea, disorientation etc.

- VR as an end product for immersive in-vehicle experiences. Currently, the applicability of VR HMDs in vehicles is limited, as portability, resolution, and synchronization between the vehicle's movements and virtual content displayed on the HMD to mitigate motion sickness need to be improved (McGill et al., 2019). However, with the rapid technological advances in this domain in recent years, one can assume that VR technology might enter vehicles in the near future for application areas such as passenger experiences including work and entertainment related activities (McGill and Brewster, 2019). 
Our analysis of the researched application areas suggests opportunities for increased user studies on virtual reality usage in higher levels of vehicle automation (SAE level 3 and higher). Finally, we propose a set of research topics to be considered in future experiments addressing virtual reality in automated driving:

- Mixed traffic analyses. As decades of shared manual and automated vehicles are ahead of us, we recommend to further investigate mixed traffic scenarios from an HMI viewpoint. Cooperative and teleoperated driving concepts and interfaces should therefore be investigated.

- Passenger experiences and interaction. As active drivers transition towards passive passengers as vehicle automation increases, work and entertainment activities (NDRTs) should be evaluated further. The role of simulator and motion sickness and its part in HMI design should be investigated as well. The advancements of $\mathrm{AR}$, and the possibility to simulate AR in VR environments, further urge researchers to explore personalization concepts, such as AR head-up and windshield displays. Additionally, topics in context awareness and multimodal interaction require more research efforts for higher levels of vehicle automation.

- Vulnerable road users. When the driver is out of the loop, and the vehicle takes over the driving task, the inclusion of pedestrians, cyclists, among other vulnerable road users, must be considered accordingly. Trust, acceptance, and ethics must be evaluated not just from the driver perspective, but with consideration of VRUs as well.

- Advancing AR simulations within VR environments. While a number of studies was carried out simulating augmented reality head-up and windshield displays in $\mathrm{VR}$, we encourage researchers to enhance the quality and realism of AR simulations within VREs, including lighting, color blending, reflections, and motion parallax.

- Outdoor studies with VR. Currently, most studies on VR in automated driving are conducted in indoor environments because of safety and technology concerns. As VR technology advances, we suggest to increasingly conduct mixed reality studies in real vehicles to help increase the external validity of these user studies.

\section{REFERENCES}

Ackermans, S., Dey, D., Ruijten, P., Cuijpers, R. H., and Pfleging, B. (2020). "The Effects of Explicit Intention Communication, Conspicuous Sensors, and Pedestrian Attitude in Interactions with Automated Vehicles," in Proceedings of the 2020 CHI Conference on Human Factors in Computing Systems, New York, NY, USA (New York, NY: Association for Computing Machinery), 1-14. CHI'20. doi:10.1145/3313831.3376197

Ali, A., Elnaggarz, A., Reichardtz, D., and Abdennadher, S. (2016). "Gamified Virtual Reality Driving Simulator for Asserting Driving Behaviors," in 2016 1st International Conference on Game, Game Art, and Gamification (ICGGAG) (IEEE), 1-6. doi:10.1109/ICGGAG.2016.8052668

Amersfoorth, E. v., Roefs, L., Bonekamp, Q., Schuermans, L., and Pfleging, B. (2019). "Increasing Driver Awareness through Translucency on Windshield

\subsection{Final Thoughts and Future Plans}

The overarching aim of this survey was to provide a comprehensive review of virtual reality usage and applications for vehicles with different levels of automation over the last decade. This work is intended to inspire researchers and practitioners in certain automotive virtual reality application areas when creating their own research plans. Further, we intended to show the historic development from low-immersive monitor/HUD setups to more advanced CAVE/ HMD setups, and simulating continuous-depth AR content within VR environments. Additionally, we aggregated methodological approaches, pointed out their limitations, and gave recommendations for future research. In the future, we intend to investigate each individual application area in more depth (e.g., virtual reality driving simulators for HCI research), and compile more detailed and focused reviews. Moreover, there is a need to constantly keep the analysis up-to-date with each finished publication year, starting with 2021. VR in automated driving is still a developing research field which needs to be observed continuously. We see that interest and efforts in this field are advancing, and we look forward to further work.

\section{DATA AVAILABILITY STATEMENT}

The original contributions presented in the study are included in the article/Supplementary Material, further inquiries can be directed to the corresponding author.

\section{AUTHOR CONTRIBUTIONS}

All authors contributed to the entire review process and the manuscript. AR1 initiated the process with database search, data collection, and analysis. AR1, AR2, and $\mathrm{CH}$ all reviewed and collected data. AR1 took the lead in writing the manuscript. We applied the SDC approach to arrange the sequence of authors.

\section{FUNDING}

This work was supported by the University of Applied Sciences $\mathrm{PhD}$ program and research subsidies granted by the government of Upper Austria.

Displays," in Proceedings of the 11th International Conference on Automotive User Interfaces and Interactive Vehicular Applications (New York, NY: Adjunct Proceedings), 156-160. doi:10.1145/3349263.3351911

Asha, A. Z., Anzum, F., Finn, P., Sharlin, E., and Costa Sousa, M. (2020). "Designing External Automotive Displays: VR Prototypes and Analysis," in 12th International Conference on Automotive User Interfaces and Interactive Vehicular Applications, 74-82. doi:10.1145/3409120.3410658

Automotive UI (2009). "Proceedings of the 1st International Conference on Automotive User Interfaces and Interactive Vehicular Applications - AutomotiveUI'09," $P$ in roceedings of the 1st International Conference on Automotive User Interfaces and Interactive Vehicular Applications, New York, NY, USA (New York, NY: Association for Computing Machinery). doi:10.1145/1620509

Ayres, G., and Mehmood, R. (2009). "On Discovering Road Traffic Information Using Virtual Reality Simulations,” in 2009 11th International Conference on Computer Modelling and Simulation, 411-416. doi:10.1109/uksim.2009.141 
Azuma, R. T. (1997). A Survey of Augmented Reality. Presence: Teleoperators \& Virtual Environments 6, 355-385. doi:10.1162/pres.1997.6.4.355

Barić, D., Havârneanu, G. M., and Măirean, C. (2020). Attitudes of Learner Drivers toward Safety at Level Crossings: Do They Change after a $360^{\circ}$ Video-Based Educational Intervention? Transportation Res. F: Traffic Psychol. Behav. 69, 335-348. doi:10.1016/j.trf.2020.01.018

Benz, T. M., Riedl, B., and Chuang, L. L. (2019). "Projection Displays Induce Less Simulator Sickness Than Head-Mounted Displays in a Real Vehicle Driving Simulator," in Proceedings of the 11th International Conference on Automotive User Interfaces and Interactive Vehicular Applications, 379-387. doi:10.1145/ 3342197.3344515

Blissing, B., Bruzelius, F., and Eriksson, O. (2019). Driver Behavior in Mixed and Virtual Reality - A Comparative Study. Transportation Res. Part F: Traffic Psychol. Behav. 61, 229-237. doi:10.1016/j.trf.2017.08.005

Blissing, B., Bruzelius, F., and Eriksson, O. (2016). Effects of Visual Latency on Vehicle Driving Behavior. ACM Trans. Appl. Percept. 14, 1-12. doi:10.1145/ 2971320

Böckle, M.-P., Brenden, A. P., Klingegård, M., Habibovic, A., and Bout, M. (2017). "SAV2P," in Proceedings of the 9th International Conference on Automotive User Interfaces and Interactive Vehicular Applications Adjunct, 136-140. doi:10.1145/3131726.3131765

Bolder, A., Grünvogel, S. M., and Angelescu, E. (2018). "Comparison of the Usability of a Car Infotainment System in a Mixed Reality Environment and in a Real Car," in Proceedings of the 24th ACM Symposium on Virtual Reality Software and Technology, 1-10. doi:10.1145/ 3281505.3281512

Bolton, A., Burnett, G., and Large, D. R. (2015). "An Investigation of Augmented Reality Presentations of Landmark-Based Navigation Using a Head-Up Display," in Proceedings of the 7th International Conference on Automotive User Interfaces and Interactive Vehicular Applications, 56-63. doi:10.1145/ 2799250.2799253

Bottone, M., and Johnsen, K. (2016). "Improving Interaction in HMD-Based Vehicle Simulators through Real Time Object Reconstruction," in Proceedings of the 2016 Symposium on Spatial User Interaction, 111-120. doi:10.1145/ 2983310.2985761

Bout, M., Brenden, A. P., Klingegård, M., Habibovic, A., and Böckle, M.-P. (2017). "A Head-Mounted Display to Support Teleoperations of Shared Automated Vehicles," in Proceedings of the 9th International Conference on Automotive User Interfaces and Interactive Vehicular Applications Adjunct, 62-66. doi: $10.1145 / 3131726.3131758$

Bowman, D. A., and McMahan, R. P. (2007). Virtual Reality: How Much Immersion Is Enough? Computer 40, 36-43. doi:10.1109/mc.2007.257

Bozkir, E., Geisler, D., and Kasneci, E. (2019). "Assessment of Driver Attention during a Safety Critical Situation in VR to Generate VR-Based Training," in ACM Symposium on Applied Perception 2019, 1-5. doi:10.1145/ 3343036.3343138

Bram-Larbi, K. F., Charissis, V., Khan, S., Lagoo, R., Harrison, D. K., and Drikakis, D. (2020). "Collision Avoidance Head-Up Display: Design Considerations for Emergency Services' Vehicles," in 2020 IEEE International Conference on Consumer Electronics (New York, NY: ICCE), 1-7. doi:10.1109/ icce46568.2020.9043068

Brand, D., Büchele, K., and Meschtscherjakov, A. (2016). "Pointing at the HUD," in Adjunct Proceedings of the 8th International Conference on Automotive User Interfaces and Interactive Vehicular Applications, 167-172. doi:10.1145/ 3004323.3004343

Brown, B., Park, D., Sheehan, B., Shikoff, S., Solomon, J., Yang, J., and Kim, I. (2018). "Assessment of Human Driver Safety at Dilemma Zones with Automated Vehicles through a Virtual Reality Environment," in 2018 Systems and Information Engineering Design Symposium (SIEDS)IEEE, 185-190. doi:10.1109/sieds.2018.8374733

Calvi, A., D'Amico, F., Ferrante, C., and Bianchini Ciampoli, L. (2020a). Effectiveness of Augmented Reality Warnings on Driving Behaviour whilst Approaching Pedestrian Crossings: A Driving Simulator Study. Accid. Anal. Prev. 147, 105760. doi:10.1016/j.aap.2020.105760

Calvi, A., D'Amico, F., Ferrante, C., and Bianchini Ciampoli, L. (2020b). Evaluation of Augmented Reality Cues to Improve the Safety of Left-Turn Maneuvers in a Connected Environment: A Driving Simulator Study. Accid. Anal. Prev. 148, 105793. doi:10.1016/j.aap.2020.105793
Chang, C.-M., Toda, K., Sakamoto, D., and Igarashi, T. (2017). “Eyes on a Car," in Proceedings of the 9th international conference on automotive user interfaces and interactive vehicular applications, 65-73. doi:10.1145/3122986.3122989

Charissis, V. (2014). "Enhancing Human Responses through Augmented Reality Head-Up Display in Vehicular Environment," in 2014 11th International Conference \& Expo on Emerging Technologies for a Smarter World (CEWIT) (IEEE), 1-6. doi:10.1109/cewit.2014.7021141

Charissis, V., Papanastasiou, S., Chan, W., and Peytchev, E. (2013). "Evolution of a Full-Windshield HUD Designed for Current VANET Communication Standards," in 2013 16th International IEEE Conference on Intelligent Transportation Systems ((ITSC) (IEEE)), 1637-1643. doi:10.1109/ itsc.2013.6728464

Chen, K. B., Xu, X., Lin, J.-H., and Radwin, R. G. (2015). Evaluation of Older Driver Head Functional Range of Motion Using Portable Immersive Virtual Reality. Exp. Gerontol. 70, 150-156. doi:10.1016/j.exger.2015.08.010

Chia, A., and Balash, C. (2020). Towards an Improved Intuitive Interface for Remotely Operated Vehicles. J. Ocean Eng. Sci. 5, 346-357. doi:10.1016/ j.joes.2020.03.001

Colley, M., Bräuner, C., Lanzer, M., Walch, M., Baumann, M., and Rukzio, E. (2020). "Effect of Visualization of Pedestrian Intention Recognition on Trust and Cognitive Load," in 12th International Conference on Automotive User Interfaces and Interactive Vehicular Applications, 181-191. doi:10.1145/ 3409120.3410648

Daimler, A. G. (2020). Meet the S-Class Digital: "my Mbux". Available at: https:// bit.ly/32fu9RU. (Accessed: 2020-08-20) Dataset.

de Clercq, K., Dietrich, A., Núñez Velasco, J. P., de Winter, J., and Happee, R. (2019). External Human-Machine Interfaces on Automated Vehicles: Effects on Pedestrian Crossing Decisions. Hum. Factors 61, 1353-1370. doi:10.1177/ 0018720819836343

De Winter, J., van Leeuwen, P. M., and Happee, R. (2012). "Advantages and Disadvantages of Driving Simulators: A Discussion," in Proceedings of measuring behavior

Deb, S., Strawderman, L. J., and Carruth, D. W. (2018). Investigating Pedestrian Suggestions for External Features on Fully Autonomous Vehicles: A Virtual Reality experiment. Transportation Res. Part F: Traffic Psychol. Behav. 59, 135-149. doi:10.1016/j.trf.2018.08.016

Detjen, H., Geisler, S., and Schneegass, S. (2020). ““Help, Accident Ahead!”: Using Mixed Reality Environments in Automated Vehicles to Support Occupants after Passive Accident Experiences," in 12th International Conference on Automotive User Interfaces and Interactive Vehicular Applications, 58-61. doi:10.1145/3409251.3411723

Dey, A., Billinghurst, M., Lindeman, R. W., and Swan, J. E. (2018). A Systematic Review of 10 Years of Augmented Reality Usability Studies: 2005 to 2014. Front. Robot. AI 5, 37. doi:10.3389/frobt.2018.00037

Djavadian, S., Farooq, B., Vasquez, R., and Yip, G. (2020). "Virtual Immersive Reality Based Analysis of Behavioural Responses in Connected and Autonomous Vehicle Environment," in Mapping the Travel Behavior Genome (Elsevier), 543-559. doi:10.1016/b978-0-12-817340-4.00027-9

Doric, I., Frison, A.-K., Wintersberger, P., Riener, A., Wittmann, S., Zimmermann, M., et al. (2016). "A Novel Approach for Researching Crossing Behavior and Risk Acceptance," in Adjunct proceedings of the 8th international conference on automotive user interfaces and interactive vehicular applications, 39-44. doi: $10.1145 / 3004323.3004324$

Feierle, A., Beller, D., and Bengler, K. (2019). "Head-Up Displays in Urban Partially Automated Driving: Effects of Using Augmented Reality," in 2019 IEEE Intelligent Transportation Systems Conference (ITSC) (IEEE), 1877-1882. doi:10.1109/itsc.2019.8917472

Feldstein, I. T., and Dyszak, G. N. (2020). Road Crossing Decisions in Real and Virtual Environments: A Comparative Study on Simulator Validity. Accid. Anal. Prev. 137, 105356. doi:10.1016/j.aap.2019.105356

Ferrier-Barbut, E., Vaufreydaz, D., David, J.-A., Lussereau, J., and Spalanzani, A. (2018). "Personal Space of Autonomous Car's Passengers Sitting in the Driver's Seat," in 2018 IEEE Intelligent Vehicles Symposium (IV) (IEEE). 2022-2029. doi:10.1109/ivs.2018.8500648

Forster, Y., Hergeth, S., Naujoks, F., Beggiato, M., Krems, J. F., and Keinath, A. (2019). Learning to Use Automation: Behavioral Changes in Interaction with Automated Driving Systems. Transportation Res. F: traffic Psychol. Behav. 62, 599-614. doi:10.1016/j.trf.2019.02.013 
Frémont, V., Phan, M.-T., and Thouvenin, I. (2019). Adaptive Visual Assistance System for Enhancing the Driver Awareness of Pedestrians. Int. J. HumanComputer Interaction 36, 856-869. doi:10.1080/10447318.2019.1698220

Frison, A.-K., Forster, Y., Wintersberger, P., Geisel, V., and Riener, A. (2020). Where We Come from and where We Are Going: A Systematic Review of Human Factors Research in Driving Automation. Appl. Sci. 10, 8914. doi:10.3390/app10248914

Frison, A.-K., Wintersberger, P., Riener, A., and Schartmüller, C. (2017). "Driving Hotzenplotz: A Hybrid Interface for Vehicle Control Aiming to Maximize Pleasure in Highway Driving," in Proceedings of the 9th international conference on automotive user interfaces and interactive vehicular applications, 236-244.

Frison, A.-K., Wintersberger, P., Schartmüller, C., and Riener, A. (2019). "The Real $\mathrm{T}(\mathrm{H})$ or: Evaluation of Emergency Take-Over on a Test Track," in Proceedings of the 11th international conference on automotive user interfaces and interactive vehicular applications: Adjunct proceedings, 478-482.

Gabbard, J. L., Fitch, G. M., and Kim, H. (2014). Behind the Glass: Driver Challenges and Opportunities for AR Automotive Applications. Proc. IEEE 102, 124-136. doi:10.1109/jproc.2013.2294642

Gabbard, J. L., Smith, M., Tanous, K., Kim, H., and Jonas, B. (2019). AR DriveSim: An Immersive Driving Simulator for Augmented Reality Head-Up Display Research. Front. Robot. AI 6, 98. doi:10.3389/frobt.2019.00098

Gartner, Inc (2014). Gartner Hype Cycle for Emerging Technologies. Available at: https://www.gartner.com/en/documents/2809728/hype-cycle-for-emergingtechnologies-2014. (Accessed: 2020-05-07 [Dataset]

Gartner, Inc (2019). Gartner Hype Cycle for Emerging Technologies. Available at: https://www.gartner.com/en/documents/3956015/hype-cycle-for-emergingtechnologies-2019. (Accessed: 2020-05-07 [Dataset]

Gartner, Inc. (2021). Interpreting Technology Hype. Available at: https://www. gartner.com/en/research/methodologies/gartner-hype-cycle. (Accessed: 202005-07) [Dataset]

GENIVI Vehicle Simulator (2016). GENIVI Vehicle Simulator. Available at: https://at.projects.genivi.org/wiki/display/PROJ/GENIVI+Vehicle+Simulator. (Accessed: 2021-01-30) [Dataset]

Gerber, M. A., Schroeter, R., and Vehns, J. (2019). “A Video-Based Automated Driving Simulator for Automotive UI Prototyping, UX and Behaviour Research," in Proceedings of the 11th International Conference on Automotive User Interfaces and Interactive Vehicular Applications, 14-23. doi:10.1145/3342197.3344533

Gerber, M. A., Schroeter, R., Xiaomeng, L., and Elhenawy, M. (2020). "SelfInterruptions of Non-driving Related Tasks in Automated Vehicles: Mobile vs Head-Up Display," in Proceedings of the 2020 CHI Conference on Human Factors in Computing Systems, 1-9. doi:10.1145/3313831.3376751

Ghiurãu, F.-T., Baytaş, M. A., and Wickman, C. (2020). "ARCAR: On-Road Driving in Mixed Reality by Volvo Cars," in Adjunct Publication of the 33rd Annual ACM Symposium on User Interface Software and Technology, 62-64. doi:10.1145/3379350.3416186

Goedicke, D., Li, J., Evers, V., and Ju, W. (2018). "VR-OOM," in Proceedings of the 2018 CHI Conference on Human Factors in Computing Systems, 1-11. doi:10.1145/3173574.3173739

Gong, L., Söderlund, H., Bogojevic, L., Chen, X., Berce, A., Fast-Berglund, Å., et al. (2020). Interaction Design for Multi-User Virtual Reality Systems: An Automotive Case Study. Proced. CIRP 93, 1259-1264. doi:10.1016/j.procir.2020.04.036

Grasso, C. J., McDearmon, M. J., and Kobayashi, Y. (2010). Virtual Driving and Eco-Simulation. Proc. 2010 Spring Simulation Multiconference 1. doi:10.1145/ 1878537.1878744

Grasso, G. M., d'Italia, G., and Battiato, S. (2020). “A Flexible Virtual Environment for Autonomous Driving Agent-Human Interaction Testing," in 2020 AEIT International Conference of Electrical and Electronic Technologies for Automotive (AEIT AUTOMOTIVE)IEEE)), 1-6. doi:10.23919/ aeitautomotive 50086.2020.9307438

Gruenefeld, U., Weiß, S., Löcken, A., Virgilio, I., Kun, A. L., and Boll, S. (2019). "VRoad," in Proceedings of the 11th International Conference on Automotive User Interfaces and Interactive Vehicular Applications (New York, NY: Adjunct Proceedings), 399-404. doi:10.1145/3349263.3351511

Ha, T., Kim, S., Seo, D., and Lee, S. (2020). Effects of Explanation Types and Perceived Risk on Trust in Autonomous Vehicles. Transportation Res. Part F: Traffic Psychol. Behav. 73, 271-280. doi:10.1016/j.trf.2020.06.021
Haeuslschmid, R., Forster, S., Vierheilig, K., Buschek, D., and Butz, A. (2017). "Recognition of Text and Shapes on a Large-Sized Head-Up Display," in Proceedings of the 2017 Conference on Designing Interactive Systems, 821-831. doi:10.1145/3064663.3064736

Haeuslschmid, R., Schnurr, L., Wagner, J., and Butz, A. (2015b). "Contact-analog Warnings on Windshield Displays Promote Monitoring the Road Scene," in Proceedings of the 7th international conference on automotive user interfaces and interactive vehicular applications, 64-71. doi:10.1145/2799250.2799274

Halabi, O., Fawal, S., Almughani, E., and Al-Homsi, L. (2017). "Driver Activity Recognition in Virtual Reality Driving Simulation," in 2017 8th International Conference on Information and Communication Systems (ICICS) (IEEE), 111-115. doi:10.1109/iacs.2017.7921955

Hanau, E., and Popescu, V. (2017). "MotionReader," in Proceedings of the 9th International Conference on Automotive User Interfaces and Interactive Vehicular Applications Adjunct, 72-76. doi:10.1145/3131726.3131741

Hannah Topliss, B., Harvey, C., and Burnett, G. (2020). "How Long Can a Driver Look? Exploring Time Thresholds to Evaluate Head-Up Display Imagery," in 12th International Conference on Automotive User Interfaces and Interactive Vehicular Applications, 9-18. doi:10.1145/3409120.3410663

Häuslschmid, R., Osterwald, S., Lang, M., and Butz, A. (2015a). "Augmenting the Driver's View with Peripheral Information on a Windshield Display," in Proceedings of the 20th International Conference on Intelligent User Interfaces, 311-321. doi:10.1145/2678025.2701393

Häuslschmid, R., Ren, D., Alt, F., Butz, A., and Höllerer, T. (2019). Personalizing Content Presentation on Large 3D Head-Up Displays. PRESENCE: Virtual and Augmented Reality 27, 80-106. doi:10.1162/pres|ltext\{\_\}al|text $\left\{\_\right\}$ 0031510.1162/pres_a_00315

Häuslschmid, R., von Bülow, M., Pfleging, B., and Butz, A. (2017). "SupportingTrust in Autonomous Driving," in Proceedings of the 22nd international conference on intelligent user interfaces, 319-329. doi:10.1145/3025171.3025198

Hensch, A.-C., Rauh, N., Schmidt, C., Hergeth, S., Naujoks, F., Krems, J. F., et al. (2020). Effects of Secondary Tasks and Display Position on Glance Behavior during Partially Automated Driving. Transportation Res. Part F: Traffic Psychol. Behav. 68, 23-32. doi:10.1016/j.trf.2019.11.014

Hettinger, L. J., and Riccio, G. E. (1992). Visually Induced Motion Sickness in Virtual Environments. Presence: Teleoperators \& Virtual Environments 1, 306-310. doi:10.1162/pres.1992.1.3.306

Hock, P., Benedikter, S., Gugenheimer, J., and Rukzio, E. (2017). "CarVR," in Proceedings of the $2017 \mathrm{CHI}$ Conference on Human Factors in Computing Systems, 4034-4044. doi:10.1145/3025453.3025665

Hock, P., Kraus, J., Walch, M., Lang, N., and Baumann, M. (2016). “Elaborating Feedback Strategies for Maintaining Automation in Highly Automated Driving," in Proceedings of the 8th international conference on automotive user interfaces and interactive vehicular applications, 105-112. doi:10.1145/ 3003715.3005414

Holdgrun, T., Doric, I., Brandmeier, T., Fuchs, T., Mihlbauer, J., Steinert, P., and Peldschus, S. (2018). "A Virtual Reality Based Approach for Researching Pedestrian to Vehicle Collisions," in 2018 IEEE Intelligent Vehicles Symposium (IV) (IEEE), 1318-1325. doi:10.1109/ivs.2018.8500524

Holländer, K., Colley, A., Mai, C., Häkkilä, J., Alt, F., and Pfleging, B. (2019a). "Investigating the Influence of External Car Displays on Pedestrians' Crossing Behavior in Virtual Reality," in Proceedings of the 21st International Conference on Human-Computer Interaction with Mobile Devices and Services, 1-11. doi:10.1145/3338286.3340138

Holländer, K., Wintersberger, P., and Butz, A. (2019b). "Overtrust in External Cues of Automated Vehicles," in Proceedings of the 11th international conference on automotive user interfaces and interactive vehicular applications, 211-221. doi:10.1145/3342197.3344528

Howard, A., and Borenstein, J. (2018). The Ugly Truth about Ourselves and Our Robot Creations: the Problem of Bias and Social Inequity. Sci. Eng. Ethics 24 1521-1536. doi:10.1007/s11948-017-9975-2

Hudson, C. R., Deb, S., Carruth, D. W., McGinley, J., and Frey, D. (2018). "Pedestrian Perception of Autonomous Vehicles with External Interacting Features," in International Conference on Applied Human Factors and Ergonomics (Springer), 33-39. doi:10.1007/978-3-319-94334-3_5

Hwang, Y., Park, B.-J., and Kim, K.-H. (2016). The Effects of Augmented-Reality Head-Up Display System Usage on Drivers? Risk Perception and Psychological Change. Etri J. 38, 757-766. doi:10.4218/etrij.16.0115.0770 
Ihemedu-Steinke, Q. C., Erbach, R., Halady, P., Meixner, G., and Weber, M. (2017). "Virtual Reality Driving Simulator Based on Head-Mounted Displays," in Automotive User Interfaces (Springer), 401-428. doi:10.1007/978-3-31949448-7_15

Inners, M., and Kun, A. L. (2017). "Beyond Liability: Legal Issues of HumanMachine Interaction for Automated Vehicles," in Proceedings of the 9th International Conference on Automotive User Interfaces and Interactive Vehicular Applications, 245-253.

ISO (2018). ISO 9241-11 Ergonomics of Human-System Interaction - Part 11: Usability: Definitions and Concepts. Dataset.

Jakus, G., Dicke, C., and Sodnik, J. (2014). A User Study of Auditory, Head-Up and Multi-Modal Displays in Vehicles. Appl. Ergon. 46 Pt A, 184-192. doi:10.1016/ j.apergo.2014.08.008 A,

Jayaraman, S. K., Creech, C., Robert Jr., L. P., Tilbury, D. M., Yang, X. J., Pradhan, A. K., and Tsui, K. M. (2018). "Trust in AV," in Companion of the 2018 ACM/ IEEE international conference on human-robot interaction, 133-134. doi: $10.1145 / 3173386.3177073$

Jinjakam, C., and Hamamoto, K. (2013). "Parallax, Position and Height Difference Effects on Simulator Sickness in Immersive Virtual Environment," in The 6th 2013 Biomedical Engineering International Conference (IEEE), 1-4. doi:10.1109/bmeicon.2013.668771710.1109/bmeicon.2013.6687717

Jose, R., Lee, G. A., and Billinghurst, M. (2016). "A Comparative Study of Simulated Augmented Reality Displays for Vehicle Navigation," in Proceedings of the 28th Australian conference on computer-human interaction, 40-48. doi:10.1145/ 3010915.3010918

Karatas, N., Tanaka, T., Fujikakc, K., Yoshihara, Y., Kanamori, H., Fuwamoto, Y., and Yoshida, M. (2020). "Evaluation of AR-HUD Interface during an Automated Intervention in Manual Driving," in 2020 IEEE Intelligent Vehicles Symposium (IV), 2158-2164. doi:10.1109/iv47402.2020.9304610

Karl, I., Berg, G., Ruger, F., and Farber, B. (2013). Driving Behavior and Simulator Sickness while Driving the Vehicle in the Loop: Validation of Longitudinal Driving Behavior. IEEE Intell. Transport. Syst. Mag. 5, 42-57. doi:10.1109/ mits.2012.2217995

Kaß, C., Schoch, S., Naujoks, F., Hergeth, S., Keinath, A., and Neukum, A. (2020). Standardized Test Procedure for External Human-Machine Interfaces of Automated Vehicles. Information 11, 173. doi:10.3390/info11030173

Kennedy, R. S., and Fowlkes, J. E. (1992). Simulator Sickness Is Polygenic and Polysymptomatic: Implications for Research. Int. J. Aviation Psychol. 2, 23-38. doi:10.1207/s15327108ijap0201_2

Kim, H., Wu, X., Gabbard, J. L., and Polys, N. F. (2013). "Exploring Head-Up Augmented Reality Interfaces for Crash Warning Systems," in Proceedings of the 5th International Conference on Automotive User Interfaces and Interactive Vehicular Applications, 224-227. doi:10.1145/2516540.2516566

Kim, S., and Dey, A. K. (2009). "Simulated Augmented Reality Windshield Display as a Cognitive Mapping Aid for Elder Driver Navigation," in Proceedings of the SIGCHI Conference on Human Factors in Computing Systems. 133. doi:10.1145/1518701.1518724

Kodama, R., Koge, M., Taguchi, S., and Kajimoto, H. (2017). "COMS-VR: Mobile Virtual Reality Entertainment System Using Electric Car and Head-Mounted Display," in 2017 IEEE symposium on 3D user interfaces (3DUI) (IEEE), 130-133. doi:10.1109/3dui.2017.7893329

Koilias, A., Mousas, C., Rekabdar, B., and Anagnostopoulos, C.-N. (2019). "Passenger Anxiety when Seated in a Virtual Reality Self-Driving Car," in 2019 IEEE Conference on Virtual Reality and 3D User Interfaces (VR) (IEEE), 1024-1025. doi:10.1109/vr.2019.8798084

Konkol, K., Brandenburg, E., Stark, R., and Stark, R. (2020). "Modular Virtual Reality to Enable Efficient User Studies for Autonomous Driving," in Proceedings of the 13th ACM International Conference on PErvasive Technologies Related to Assistive Environments, 1-2. doi:10.1145/ 3389189.3397647

Kraft, A.-K., Maag, C., and Baumann, M. (2020a). Comparing Dynamic and Static Illustration of an HMI for Cooperative Driving. Accid. Anal. Prev. 144, 105682. doi:10.1016/j.aap.2020.105682

Kraft, A.-K., Maag, C., Cruz, M. I., Baumann, M., and Neukum, A. (2020b). The Effect of Visual HMIs of a System Assisting Manual Drivers in Manoeuvre Coordination in System Limit and System Failure Situations. Transportation Res. Part F: Traffic Psychol. Behav. 74, 81-94. doi:10.1016/j.trf.2020.08.002
Kun, A. L., Meulen, H. v. d., and Janssen, C. P. (2019). Calling while Driving Using Augmented Reality: Blessing or Curse?. PRESENCE: Virtual and Augmented Reality 27, 1-14. doi:10.1162/pres_a_00316

Lagoo, R., Charissis, V., Chan, W., Khan, S., and Harrison, D. (2018). "Prototype Gesture Recognition Interface for Vehicular Head-Up Display System," in 2018 IEEE International Conference on Consumer Electronics (New York, NY: ICCE), 1-6. doi:10.1109/icce.2018.8326146

Lagoo, R., Charissis, V., and Harrison, D. K. (2019). Mitigating Driver's Distraction: Automotive Head-Up Display and Gesture Recognition System. IEEE Consumer Electron. Mag. 8, 79-85. doi:10.1109/mce.2019.2923896

Lakier, M., Nacke, L. E., Igarashi, T., Vogel, D., Lakier, M., Nacke, L. E., et al. (2019). "Cross-Car, Multiplayer Games for Semi-autonomous Driving," in Proceedings of the Annual Symposium on Computer-Human Interaction in Play, 467-480. doi: $10.1145 / 3311350.3347166$

Langlois, S., and Soualmi, B. (2016). "Augmented Reality versus Classical HUD to Take over from Automated Driving: An Aid to Smooth Reactions and to Anticipate Maneuvers," in 2016 IEEE 19th international conference on intelligent transportation systems (ITSC) (IEEE), 1571-1578. doi:10.1109/ itsc.2016.7795767

Langlois, S., That, T. N., and Mermillod, P. (2016). "Virtual Head-Up Displays for Augmented Reality in Cars," in Proceedings of the European Conference on Cognitive Ergonomics, 1-8. doi:10.1145/2970930.2970946

Lee, J., and Moray, N. (1992). Trust, Control Strategies and Allocation of Function in Human-Machine Systems. Ergonomics 35, 1243-1270. doi:10.1080/ 00140139208967392

Lee, S. C., Nadri, C., Sanghavi, H., and Jeon, M. (2020). "Exploring User Needs and Design Requirements in Fully Automated Vehicles," in Extended Abstracts of the 2020 CHI Conference on Human Factors in Computing Systems, 1-9. doi: $10.1145 / 3334480.3382881$

Li, J., George, C., Ngao, A., Holländer, K., Mayer, S., and Butz, A. (2020a). “An Exploration of Users' Thoughts on Rear-Seat Productivity in Virtual Reality," in 12th International Conference on Automotive User Interfaces and Interactive Vehicular Applications, 92-95. doi:10.1145/3409251.3411732

Li, X., Schroeter, R., Rakotonirainy, A., Kuo, J., and Lenné, M. G. (2020b). Effects of Different Non-driving-related-task Display Modes on Drivers' Eye-Movement Patterns during Take-Over in an Automated Vehicle. Transportation Res. Part F: Traffic Psychol. Behav. 70, 135-148. doi:10.1016/j.trf.2020.03.001

Likitweerawong, K., and Palee, P. (2018). "The Virtual Reality Serious Game for Learning Driving Skills before Taking Practical Test," in 2018 International Conference on Digital Arts (New York, NY: Media and Technology (ICDAMT)), 158-161. doi:10.1109/icdamt.2018.8376515

Lindemann, P., Lee, T.-Y., and Rigoll, G. (2018a). "An Explanatory Windshield Display Interface with Augmented Reality Elements for Urban Autonomous Driving," in 2018 IEEE International Symposium on Mixed and Augmented Reality Adjunct (ISMAR-Adjunct)IEEE), 36-37. doi:10.1109/ ismar.adjunct.2018.00027

Lindemann, P., Lee, T.-Y., and Rigoll, G. (2018b). Catch My Drift: Elevating Situation Awareness for Highly Automated Driving with an Explanatory Windshield Display User Interface. Mti 2, 71. doi:10.3390/mti2040071

Lindemann, P., Muller, N., and Rigolll, G. (2019). "Exploring the Use of Augmented Reality Interfaces for Driver Assistance in Short-Notice Takeovers," in 2019 IEEE Intelligent Vehicles Symposium (IV) (IEEE), 804-809. doi:10.1109/ivs.2019.8814237

Lindemann, P., and Rigoll, G. (2017). "A Diminished Reality Simulation for Driver-Car Interaction with Transparent Cockpits," in 2017 IEEE Virtual Reality (VR) (IEEE), 305-306. doi:10.1109/vr.2017.7892298

Löcken, A., Golling, C., and Riener, A. (2019). "How Should Automated Vehicles Interact with Pedestrians?," in Proceedings of the 11th international conference on automotive user interfaces and interactive vehicular applications, 262-274. doi:10.1145/3342197.3344544

Lorenz, L., Kerschbaum, P., and Schumann, J. (2014). "Designing Take over Scenarios for Automated Driving," in Proceedings of the Human Factors and Ergonomics Society Annual Meeting, Los Angeles, CA (Thousand Oaks, CAMcGi: SAGE Publications Sage CA), 58. 1681-1685. doi:10.1177/ 1541931214581351

Lucas, G., Kemeny, A., Paillot, D., and Colombet, F. (2020). A Simulation Sickness Study on a Driving Simulator Equipped with a Vibration Platform. 
Transportation Res. Part F: Traffic Psychol. Behav. 68, 15-22. doi:10.1016/ j.trf.2019.11.011

Luo, M., Kamel, A. E., and Gong, G. (2012). Simulation of Natural Environment Impacts on Intelligent Vehicle Based on a Virtual Reality Platform. IFAC Proc. 45, 116-121. doi:10.3182/20120912-3-bg-2031.00022

Mallaro, S., Rahimian, P., O’Neal, E. E., Plumert, J. M., and Kearney, J. K. (2017). “A Comparison of Head-Mounted Displays vs. Large-Screen Displays for an Interactive Pedestrian Simulator," in Proceedings of the 23rd ACM Symposium on Virtual Reality Software and Technology, 1-4. doi:10.1145/ 3139131.3139171

Manawadu, U. E., Kamezaki, M., Ishikawa, M., Kawano, T., and Sugano, S. (2016). "A Hand Gesture Based Driver-Vehicle Interface to Control Lateral and Longitudinal Motions of an Autonomous Vehicle," in 2016 IEEE International Conference on Systems, Man, and Cybernetics (SMC) (IEEE), 001785-001790. doi:10.1109/smc.2016.7844497

Maruhn, P., Dietrich, A., Prasch, L., and Schneider, S. (2020). "Analyzing Pedestrian Behavior in Augmented Reality - Proof of Concept," in 2020 IEEE Conference on Virtual Reality and 3D User Interfaces (VR) (IEEE), 313-321. doi:10.1109/vr46266.2020.1581242905378

McGill, M., and Brewster, S. (2019). "Virtual Reality Passenger Experiences," in Proceedings of the 11th International Conference on Automotive User Interfaces and Interactive Vehicular Applications (New York, NY: Adjunct Proceedings), 434-441. doi:10.1145/3349263.3351330

McGill, M., Ng, A., and Brewster, S. (2017). "I Am the Passenger," in Proceedings of the 2017 chi conference on human factors in computing systems, 5655-5668. doi:10.1145/3025453.3026046

McGill, M., Williamson, J., Ng, A., Pollick, F., and Brewster, S. (2019). Challenges in Passenger Use of Mixed Reality Headsets in Cars and Other Transportation. Virtual Reality 24, 583-603. doi:10.1007/s10055-019-00420-x

Medenica, Z., Kun, A. L., Paek, T., and Palinko, O. (2011). "Augmented Reality vs. Street Views," in Proceedings of the 13th International Conference on Human Computer Interaction with Mobile Devices and Services, 265. doi:10.1145/ 2037373.2037414

Merenda, C., Kim, H., Gabbard, J. L., Leong, S., Large, D. R., and Burnett, G. (2017). "Did You See Me?," in Proceedings of the 9th International Conference on Automotive User Interfaces and Interactive Vehicular Applications, 40-49. doi:10.1145/3122986.3123013

Merenda, C., Suga, C., Gabbard, J. L., and Misu, T. (2019b). "Effects of "RealWorld" Visual Fidelity on AR Interface Assessment: A Case Study Using AR Head-Up Display Graphics in Driving," in 2019 IEEE International Symposium on Mixed and Augmented Reality (ISMAR)IEEE). 145-156. doi:10.1109/ ismar.2019.00-10

Merenda, C., Suga, C., Gabbard, J., and Misu, T. (2019a). "Effects of Vehicle Simulation Visual Fidelity on Assessing Driver Performance and Behavior," in 2019 IEEE Intelligent Vehicles Symposium (IV) (IEEE), 1679-1686. doi:10.1109/ivs.2019.8813863

Milgram, P., Takemura, H., Utsumi, A., and Kishino, F. (1995). "Augmented Reality: A Class of Displays on the Reality-Virtuality Continuum," in Telemanipulator and Telepresence Technologies (Bellingham, WA: International Society for Optics and Photonics), 2351, 282-292.

Moehring, M., and Froehlich, B. (2011). Natural Interaction Metaphors for Functional Validations of Virtual Car Models. IEEE Trans. Vis. Comput. Graphics 17, 1195-1208. doi:10.1109/tvcg.2011.36

Moher, D., Liberati, A., Tetzlaff, J., Altman, D. G., et al. (2010). Preferred Reporting Items for Systematic Reviews and Meta-Analyses: the Prisma Statement. Int. J. Surg. 8, 336-341. doi:10.1016/j.ijsu.2010.02.007

Morley, D. C., Lawrence, G., and Smith, S. (2016). "Virtual Reality User Experience as a Deterrent for Smartphone Use while Driving," in Proceedings of the 9th ACM International Conference on PErvasive Technologies Related to Assistive Environments, 1-3. doi:10.1145/2910674.2910696

Morra, L., Lamberti, F., Prattico, F. G., Rosa, S. L., and Montuschi, P. (2019). Building Trust in Autonomous Vehicles: Role of Virtual Reality Driving Simulators in HMI Design. IEEE Trans. Veh. Technol. 68, 9438-9450. doi:10.1109/tvt.2019.2933601

Mortimer, M., Eugene, A., Thomson, S., Seyedmahmoudian, M., and Horan, B. (2019). "Hector-VR: A Mixed-Reality Driving Simulator for Older Drivers," in 2019 14th Annual Conference System of Systems Engineering (SoSE) (IEEE), 200-205. doi:10.1109/sysose.2019.8753836
Neumeier, S., Höpp, M., and Facchi, C. (2019). "Yet Another Driving Simulator OpenROUTS3D: The Driving Simulator for Teleoperated Driving," in 2019 IEEE International Conference on Connected Vehicles and Expo (ICCVE) (IEEE), 1-6. doi:10.1109/iccve45908.2019.8965037

Nguyen, T. T., Holländer, K., Hoggenmueller, M., Parker, C., and Tomitsch, M. (2019). "Designing for Projection-Based Communication between Autonomous Vehicles and Pedestrians," in Proceedings of the 11th international conference on automotive user interfaces and interactive vehicular applications, 284-294. doi:10.1145/3342197.3344543

Norman, D. A. (2010). Natural User Interfaces Are Not Natural. interactions 17, 6-10. doi:10.1145/1744161.1744163

Nuñez Velasco, J. P., Farah, H., van Arem, B., and Hagenzieker, M. P. (2019). Studying Pedestrians' Crossing Behavior when Interacting with Automated Vehicles Using Virtual Reality. Transportation Res. Part F: Traffic Psychol. Behav. 66, 1-14. doi:10.1016/j.trf.2019.08.015

Otherson, I., Conti-Kufner, A. S., Dietrich, A., Maruhn, P., and Bengler, K. (2018). "Designing for Automated Vehicle and Pedestrian Communication: Perspectives on Ehmis from Older and Younger Persons," in Proceedings of the Human Factors and Ergonomics Society Europe, 135-148.

Palinko, O., Kun, A. L., Cook, Z., Downey, A., Lecomte, A., Swanson, M., and Tomaszewski, T. (2013). "Towards Augmented Reality Navigation Using Affordable Technology," in Proceedings of the 5th International Conference on Automotive User Interfaces and Interactive Vehicular Applications, 238-241. doi:10.1145/2516540.2516569

Pallamin, N., and Bossard, C. (2016). Presence, Behavioural Realism and Performances in Driving Simulation. IFAC-PapersOnLine 49, 408-413. doi:10.1016/j.ifacol.2016.10.600

Pan, X., Zhang, Z., Qu, J., Zhao, C., and Li, Y. (2017). "Enjoy Driving from Thought in a Virtual City," in 2017 36th Chinese Control Conference (CCC) (IEEE), 11034-11040. doi:10.23919/chicc.2017.8029119

Paredes, P. E., Balters, S., Qian, K., Murnane, E. L., Ordóñez, F., Ju, W., and Landay, J. A. (2018). "Driving with the Fishes," in Proceedings of the ACM on Interactive, Mobile, Wearable and Ubiquitous Technologies, 1-21. doi:10.1145/3287062Proc. ACM Interact. Mob. Wearable Ubiquitous Technol.2

Perez, D., Hasan, M., Shen, Y., and Yang, H. (2019). AR-PED: A Framework of Augmented Reality Enabled Pedestrian-In-The-Loop Simulation. Simulation Model. Pract. Theor. 94, 237-249. doi:10.1016/j.simpat.2019.03.005

Pettersson, I., Karlsson, M., and Ghiurau, F. T. (2019). "Virtually the Same Experience?," in Proceedings of the 2019 on Designing Interactive Systems Conference, 463-473. doi:10.1145/3322276.3322288

Pettersson, I., Lachner, F., Frison, A.-K., Riener, A., and Butz, A. (2018). "A bermuda triangle? a Review of Method Application and Triangulation in User Experience Evaluation," in Proceedings of the 2018 CHI Conference on Human Factors in Computing Systems, 1-16.

Pfleging, B., Rang, M., and Broy, N. (2016). "Investigating User Needs for Nondriving-related Activities during Automated Driving," in Proceedings of the 15th international conference on mobile and ubiquitous multimedia, 91-99. doi: $10.1145 / 3012709.3012735$

Phan, M. T., Thouvenin, I., and Fremont, V. (2016). "Enhancing the Driver Awareness of Pedestrian Using Augmented Reality Cues," in 2016 IEEE 19th International Conference on Intelligent Transportation Systems (ITSC) (IEEE), 1298-1304. doi:10.1109/itsc.2016.7795724

Pichen, J., Yan, F., and Baumann, M. (2020). "Towards a Cooperative DriverVehicle Interface: Enhancing Drivers' Perception of Cyclists through Augmented Reality," in 2020 IEEE Intelligent Vehicles Symposium (IV) (IEEE), 1827-1832. doi:10.1109/iv47402.2020.9304621

Prabhakar, G., Ramakrishnan, A., Madan, M., Murthy, L. R. D., Sharma, V. K., Deshmukh, S., et al. (2019). Interactive Gaze and finger Controlled HUD for Cars. J. Multimodal User Inter. 14, 101-121. doi:10.1007/s12193-019-00316-9

Pugliese, B. J., Barton, B. K., Davis, S. J., and Lopez, G. (2020). Assessing Pedestrian Safety across Modalities via a Simulated Vehicle Time-To-Arrival Task. Accid. Anal. Prev. 134, 105344. doi:10.1016/j.aap.2019.105344

Rangelova, S., and Andre, E. (2019). A Survey on Simulation Sickness in Driving Applications with Virtual Reality Head-Mounted Displays. PRESENCE: Virtual and Augmented Reality 27, 15-31. doi:10.1162/pres_a_00318

Razmi Rad, S., Homem de Almeida Correia, G., and Hagenzieker, M. (2020). Pedestrians' Road Crossing Behaviour in Front of Automated Vehicles: Results 
from a Pedestrian Simulation experiment Using Agent-Based Modelling. Transportation Res. Part F: Traffic Psychol. Behav. 69, 101-119. doi:10.1016/ j.trf.2020.01.014

Reason, J. T., and Brand, J. J. (1975). Motion Sickness. Academic Press.

Rhiu, I., Kim, Y. M., Kim, W., and Yun, M. H. (2020). The Evaluation of User Experience of a Human Walking and a Driving Simulation in the Virtual Reality. Int. J. Ind. Ergon. 79, 103002. doi:10.1016/j.ergon.2020.103002

Riegler, A., Aksoy, B., Riener, A., and Holzmann, C. (2020a). "Gaze-based Interaction with Windshield Displays for Automated Driving: Impact of Dwell Time and Feedback Design on Task Performance and Subjective Workload," in 12th International Conference on Automotive User Interfaces and Interactive Vehicular Applications, 151-160. doi:10.1145/ 3409120.3410654

Riegler, A., Riener, A., and Holzmann, C. (2020b). "A Research Agenda for Mixed Reality in Automated Vehicles," in 19th International Conference on Mobile and Ubiquitous Multimedia, 119-131. doi:10.1145/3428361.3428390

Riegler, A., Riener, A., and Holzmann, C. (2019a). AutoWSD. Proc. Mensch Comp. 2019, 853-857. doi:10.1145/3340764.3345366

Riegler, A., Riener, A., and Holzmann, C. (2019b). "Virtual Reality Driving Simulator for User Studies on Automated Driving," in Proceedings of the 11th International Conference on Automotive User Interfaces and Interactive Vehicular Applications (Adjunct Proceedings), New York, NY, 502-507. doi:10.1145/3349263.3349595

Riegler, A., Weigl, K., Riener, A., and Holzmann, C. (2020c). "StickyWSD: Investigating Content Positioning on a Windshield Display for Automated Driving," in 19th International Conference on Mobile and Ubiquitous Multimedia, 143-151. doi:10.1145/3428361.3428405

Robertson, R., and Vanlaar, W. (2008). Elderly Drivers: Future Challenges?. Accid. Anal. Prev. 40, 1982-1986. doi:10.1016/j.aap.2008.08.012

Rouchitsas, A., and Alm, H. (2019). External Human-Machine Interfaces for Autonomous Vehicle-To-Pedestrian Communication: A Review of Empirical Work. Front. Psychol. 10, 2757. doi:10.3389/fpsyg.2019.02757

Rousset, T., Bourdin, C., Goulon, C., Monnoyer, J., and Vercher, J.-L. (2015). "Does Virtual Reality Affect Visual Perception of Egocentric Distance?," in 2015 IEEE Virtual Reality (VR) (IEEE), 277-278. doi:10.1109/vr.2015.7223403

Rusch, M. L., Schall, Jr., M. C., Lee, J. D., Dawson, J. D., and Rizzo, M. (2014). Augmented Reality Cues to Assist Older Drivers with gap Estimation for LeftTurns. Accid. Anal. Prev. 71, 210-221. doi:10.1016/j.aap.2014.05.020

SAE On-Road Automated Vehicle Standards Committee (2021). Taxonomy and Definitions for Terms Related to On-Road Motor Vehicle Automated Driving Systems. Dataset.

Sakamura, Y., Tomita, A., Shishido, H., Mizunami, T., Inoue, K., Kameda, Y., Harada, E. T., and Kitahara, I. (2018). "A Virtual Boarding System of an Autonomous Vehicle for Investigating the Effect of an AR Display on Passenger Comfort," in 2018 IEEE International Symposium on Mixed and Augmented Reality Adjunct (ISMAR-Adjunct)IEEE)), 344-349. doi:10.1109/ismaradjunct.2018.00101

Samuel, S., Yahoodik, S., Yamani, Y., Valluru, K., and Fisher, D. L. (2020). Ethical Decision Making behind the Wheel - A Driving Simulator Study. Transportation Res. Interdiscip. Perspect. 5, 100147. doi:10.1016/ j.trip.2020.100147

Sawabe, T., Kanbara, M., and Hagita, N. (2017). "Diminished Reality for Acceleration Stimulus: Motion Sickness Reduction with Vection for Autonomous Driving," in 2017 IEEE Virtual Reality (VR) (IEEE), 277-278. doi:10.1109/vr.2017.7892284

Schartmüller, C., Riener, A., Wintersberger, P., and Frison, A.-K. (2018). "Workaholistic," in Proceedings of the 20th international conference on human-computer interaction with mobile devices and services, 1-12. doi:10.1145/3229434.3229459

Schroeter, R., and Gerber, M. A. (2018). "A Low-Cost VR-Based Automated Driving Simulator for Rapid Automotive UI Prototyping," in Adjunct Proceedings of the 10th International Conference on Automotive User Interfaces and Interactive Vehicular Applications, 248-251. doi:10.1145/ 3239092.3267418

Schroeter, R., and Steinberger, F. (2016). "Pokémon DRIVE: towards Increased Situational Awareness in Semi-automated Driving," in Proceedings of the 28th australian conference on computer-human interaction, 25-29. doi:10.1145/ 3010915.3010973
Schrom-Feiertag, H., Regal, G., and Schrammel, J. (2019). "Speed Experience: Assessing the Quality of Vehicle Speed Perception in Virtual Environments," in 2019 Eleventh International Conference on Quality of Multimedia Experience (QoMEX)IEEE), 1-3. doi:10.1109/qomex.2019.8743224

Schwarz, F., and Fastenmeier, W. (2017). Augmented Reality Warnings in Vehicles: Effects of Modality and Specificity on Effectiveness. Accid. Anal. Prev. 101, 55-66. doi:10.1016/j.aap.2017.01.019

Shah, S., Dey, D., Lovett, C., and Kapoor, A. (2017). "Airsim: High-Fidelity Visual and Physical Simulation for Autonomous Vehicles," in Field and Service Robotics. doi:10.1007/978-3-319-67361-5_40

Shahrdar, S., Park, C., and Nojoumian, M. (2019). "Human Trust Measurement Using an Immersive Virtual Reality Autonomous Vehicle Simulator," in Proceedings of the 2019 AAAI/ACM Conference on AI, (New York, NY: Ethics and Society), 515-520. doi:10.1145/3306618.3314264

Shahriar, S. T., and Kun, A. L. (2018). "Camera-View Augmented Reality," in Proceedings of the 10th International Conference on Automotive User Interfaces and Interactive Vehicular Applications, 146-154. doi:10.1145/ 3239060.3240447

Sibi, S., Balters, S., Fu, E., Strack, E. G., Steinert, M., and Ju, W. (2020). "Back to School: Impact of Training on Driver Behavior and State in Autonomous Vehicles," in 2020 IEEE Intelligent Vehicles Symposium (New York, NY: IV), 1189-1196. doi:10.1109/iv47402.2020.9304537

Singer, T., Kobbert, J., Zandi, B., and Khanh, T. Q. (2020). Displaying the Driving State of Automated Vehicles to Other Road Users: An International, Virtual Reality-Based Study as a First Step for the Harmonized Regulations of Novel Signaling Devices. IEEE Trans. Intell. Transport. Syst., 1, 1, 15. doi:10.1109/ tits.2020.3032777

Slater, M., Antley, A., Davison, A., Swapp, D., Guger, C., Barker, C., et al. (2006). A Virtual Reprise of the stanley Milgram Obedience Experiments. PloS one 1, e39. doi:10.1371/journal.pone.0000039

Smith, M., Gabbard, J. L., Burnett, G., and Doutcheva, N. (2017). The Effects of Augmented Reality Head-Up Displays on Drivers' Eye Scan Patterns, Performance, and Perceptions. Int. J. Mobile Hum. Comp. Interaction 9, 1-17. doi:10.4018/ijmhci.2017040101

Smith, M., Gabbard, J. L., and Conley, C. (2016). "Head-Up vs. Head-Down Displays," in Proceedings of the 8th international conference on Automotive User Interfaces and Interactive Vehicular Applications, 185-192. doi:10.1145/ 3003715.3005419

Soro, A., Rakotonirainy, A., Schroeter, R., and Wollstädter, S. (2014). "Using Augmented Video to Test In-Car User Experiences of Context Analog HUDs," in Adjunct Proceedings of the 6th International Conference on Automotive User Interfaces and Interactive Vehicular Applications, 1-6. doi:10.1145/ 2667239.2667302

Sportillo, D., Paljic, A., Boukhris, M., Fuchs, P., Ojeda, L., and Roussarie, V. (2017). "An Immersive Virtual Reality System for Semi-autonomous Driving Simulation," in Proceedings of the 9th International Conference on Computer and Automation Engineering, 6-10. doi:10.1145/3057039.3057079

Sportillo, D., Paljic, A., Ojeda, L., Fuchs, P., and Roussarie, V. (2018b). "Light Virtual Reality Systems for the Training of Conditionally Automated Vehicle Drivers," in 2018 IEEE Conference on Virtual Reality and 3D User Interfaces (VR) (IEEE), 693-694. doi:10.1109/vr.2018.8446226

Sportillo, D., Paljic, A., and Ojeda, L. (2018a). Get Ready for Automated Driving Using Virtual Reality. Accid. Anal. Prev. 118, 102-113. doi:10.1016/ j.aap.2018.06.003

Sportillo, D., Paljic, A., and Ojeda, L. (2019). "On-Road Evaluation of Autonomous Driving Training," in 2019 14th ACM/IEEE International Conference on Human-Robot Interaction (HRI) (IEEE), 182-190. doi:10.1109/ hri.2019.8673277

Stadler, S., Cornet, H., Novaes Theoto, T., and Frenkler, F. (2019). "A Tool, Not a Toy: Using Virtual Reality to Evaluate the Communication between Autonomous Vehicles and Pedestrians," in Augmented Reality and Virtual Reality (Springer), 203-216. doi:10.1007/978-3-030-06246-0_15

Steuer, J. (1992). Defining Virtual Reality: Dimensions Determining Telepresence. J. Commun. 42, 73-93. doi:10.1111/j.1460-2466.1992.tb00812.x

Stockert, S., Richardson, N. T., and Lienkamp, M. (2015). Driving in an Increasingly Automated World - Approaches to Improve the DriverAutomation Interaction. Proced. Manufacturing 3, 2889-2896. doi:10.1016/ j.promfg.2015.07.797 
Szalai, M., Varga, B., Tettamanti, T., and Tihanyi, V. (2020). "Mixed Reality Test Environment for Autonomous Cars Using Unity 3D and SUMO," in 2020 IEEE 18th World Symposium on Applied Machine Intelligence and Informatics (SAMI)IEEE)), 73-78. doi:10.1109/sami48414.2020.9108745

Taheri, S. M., Matsushita, K., and Sasaki, M. (2017a). Development of a Driving Simulator with Analyzing Driver's Characteristics Based on a Virtual Reality Head Mounted Display. JTTs 07, 351-366. doi:10.4236/jtts.2017.73023

Taheri, S. M., Matsushita, K., and Sasaki, M. (2017b). Virtual Reality Driving Simulation for Measuring Driver Behavior and Characteristics. JTTS 07, 123-132. doi:10.4236/jtts.2017.72009

Takada, S., Ueda, K., Yamada, N., Iguchi, Y., Tao, Y., Koizumi, K., et al. (2019). "Evaluation of Driver's Cognitive Load when Presented Information on the Windshield Using P300 Latency in Eye-Fixation Related Potentials," in 2019 IEEE Intelligent Transportation Systems Conference (ITSC) (IEEE)), 4006-4011. doi:10.1109/itsc.2019.8917209

Tateyama, Y., Yamada, H., Noyori, J., Mori, Y., Yamamoto, K., Ogi, T., Nishimura, H., Kitamura, N., and Yashiro, H. (2010). "Observation of Drivers' Behavior at Narrow Roads Using Immersive Car Driving Simulator," in Proceedings of the 9th ACM SIGGRAPH Conference on Virtual-Reality Continuum and its Applications in Industry. doi:10.1145/1900179.1900263391

Tesch, A., and Dörner, R. (2020). "Expert Performance in the Examination of Interior Surfaces in an Automobile: Virtual Reality vs. Reality," in Proceedings of the 28th ACM International Conference on Multimedia, 2673-2681. doi:10.1145/3394171.3413980

Thevin, L., Briant, C., and Brock, A. M. (2020). X-road. ACM Trans. Access. Comput. 13, 1-47. doi:10.1145/3377879

Thomas, F. M. F., and Charlton, S. G. (2020). Inattentional Blindness and Information Relevance of Variable Message Signs. Accid. Anal. Prev. 140, 105511. doi:10.1016/j.aap.2020.105511

Tippey, K. G., Sivaraj, E., and Ferris, T. K. (2017). Driving while Interacting with Google Glass: Investigating the Combined Effect of Head-Up Display and Hands-free Input on Driving Safety and Multitask Performance. Hum. Factors 59, 671-688. doi:10.1177/0018720817691406

Topliss, B. H., Pampel, S. M., Burnett, G., and Gabbard, J. L. (2019). "Evaluating Head-Up Displays across Windshield Locations," in Proceedings of the 11th International Conference on Automotive User Interfaces and Interactive Vehicular Applications, 244-253. doi:10.1145/3342197.3344524

Topliss, B. H., Pampel, S. M., Burnett, G., Skrypchuk, L., and Hare, C. (2018). "Establishing the Role of a Virtual Lead Vehicle as a Novel Augmented Reality Navigational Aid," in Proceedings of the 10th International Conference on Automotive User Interfaces and Interactive Vehicular Applications, 137-145. doi:10.1145/3239060.3239069

Tudor, S., Carey, S., and Dubey, R. (2015). "Development and Evaluation of a Dynamic Virtual Reality Driving Simulator," in Proceedings of the 8th ACM International Conference on PErvasive Technologies Related to Assistive Environments, 1-5. doi:10.1145/2769493.2769593

Uchida, N., Tagawa, T., and Sato, K. (2017). Development of an Augmented Reality Vehicle for Driver Performance Evaluation. IEEE Intell. Transport. Syst. Mag. 9, 35-41. doi:10.1109/mits.2016.2601943

Uijong, J., Kang, J., and Wallraven, C. (2019). You or Me? Personality Traits Predict Sacrificial Decisions in an Accident Situation. IEEE Trans. Vis. Comput. Graphics 25, 1898-1907. doi:10.1109/tvcg.2019.2899227

Ulahannan, A., Cain, R., Thompson, S., Skrypchuk, L., Mouzakitis, A., Jennings, P., et al. (2020). User Expectations of Partial Driving Automation Capabilities and Their Effect on Information Design Preferences in the Vehicle. Appl. Ergon. 82, 102969. doi:10.1016/j.apergo.2019.102969

Utsumi, A., Kawanishi, N., Nagasawa, I., Satou, K., Uchikata, K., and Hagita, N. (2018). "Impact of Directive Visual Information on Driver's Emergency Behavior," in 2018 21st International Conference on Intelligent Transportation Systems (ITSC)IEEE)), 1344-1349. doi:10.1109/ itsc.2018.8569892

Utsumi, A., Mikuni, T., and Nagasawa, I. (2019). "Effect of On-Road Virtual Visual References on Vehicle Control Stability of Wide/narrow FOV Drivers," in Proceedings of the 11th International Conference on Automotive User Interfaces and Interactive Vehicular Applications, 297-301. doi:10.1145/ 3349263.3351331Adjunct Proc.

Veronez, M. R., Gonzaga, L., Bordin, F., Kupssinsku, L., Kannenberg, G. L., Duarte, T., Santana, L. G., de Fraga, L. L., Alves, D. N., and Marson, F. P. (2018).
"RIDERS: Road Inspection \& Driver Simulation," in 2018 IEEE Conference on Virtual Reality and 3D User Interfaces (VR)IEEE)), 715-716. doi:10.1109/ vr.2018.8446207

Villalobos-Zúñiga, G., Kujala, T., and Oulasvirta, A. (2016). "T9+HUD," in Proceedings of the 8th International Conference on Automotive User Interfaces and Interactive Vehicular Applications, 177-184. doi:10.1145/ 3003715.3005453

von Sawitzky, T., Wintersberger, P., Riener, A., and Gabbard, J. L. (2019). "Increasing Trust in Fully Automated Driving," in Proceedings of the 8th ACM International Symposium on Pervasive Displays, 1-7. doi:10.1145/ 3321335.3324947

Walch, M., Frommel, J., Rogers, K., Schüssel, F., Hock, P., Dobbelstein, D., and Weber, M. (2017). "Evaluating VR Driving Simulation from a Player Experience Perspective," in Proceedings of the 2017 CHI Conference Extended Abstracts on Human Factors in Computing Systems, 2982-2989. doi:10.1145/3027063.3053202

Wang, J., Chen, J., Qiao, Y., Zhou, J., and Wang, Y. (2019a). "Online Gesture Recognition Algorithm Applied to HUD Based Smart Driving System," in 2019 IEEE International Symposium on Mixed and Augmented Reality Adjunct (ISMAR-Adjunct)IEEE)), 289-294. doi:10.1109/ismar-adjunct.2019.00-26

Wang, J., and Soffker, D. (2016). "Improving Driving Efficiency for Hybrid Electric Vehicle with Suitable Interface," in 2016 IEEE International Conference on Systems, Man, and Cybernetics (SMC) (IEEE), 000928-000933. doi:10.1109/ smc.2016.7844360

Wang, S., Charissis, V., Campbell, J., Chan, W., Moore, D., and Harrison, D. (2016). "An Investigation into the Use of Virtual Reality Technology for Passenger Infotainment in a Vehicular Environment," in 2016 International Conference on Advanced Materials for Science and Engineering (ICAMSE) IEEE)), 404-407. doi:10.1109/icamse.2016.7840359

Wang, S., Charissis, V., and Harisson, D. K. (2017). Augmented Reality Prototype HUD for Passenger Infotainment in a Vehicular Environment. Adv. Sci. Technol. Eng. Syst. J. 2, 634-641. doi:10.25046/aj020381

Wang, S., Charissis, V., Lagoo, R., Campbell, J., and Harrison, D. K. (2019b). "Reducing Driver Distraction by Utilizing Augmented Reality Head-Up Display System for Rear Passengers," in 2019 IEEE International Conference on Consumer Electronics (ICCE) (IEEE), 1-6. doi:10.1109/ icce.2019.8661927

Wang, Z., Han, K., and Tiwari, P. (2020). “Augmented Reality-Based Advanced Driver-Assistance System for Connected Vehicles," in 2020 IEEE International Conference on Systems, Man, and Cybernetics (SMC) (IEEE), 752-759. doi:10.1109/smc42975.2020.9283462

Weidner, F., and Broll, W. (2019). "Interact with Your Car: a User-Elicited Gesture Set to Inform Future In-Car User Interfaces," in Proceedings of the 18th International Conference on Mobile and Ubiquitous Multimedia. 1-12. doi: $10.1145 / 3365610.3365625$

Weidner, F., Hoesch, A., Poeschl, S., and Broll, W. (2017). "Comparing VR and Non-VR Driving Simulations: An Experimental User Study,” in 2017 IEEE Virtual Reality (VR)IEEE)), 281-282. doi:10.1109/vr.2017.7892286

Weinberg, G., Harsham, B., and Medenica, Z. (2011). "Evaluating the Usability of a Head-Up Display for Selection from Choice Lists in Cars," in Proceedings of the 3rd International Conference on Automotive User Interfaces and Interactive Vehicular Applications, 39-46. doi:10.1145/2381416.2381423

Williams, B., Garton, A. E., and Headleand, C. J. (2020). "Exploring Visuo-Haptic Feedback Congruency in Virtual Reality," in 2020 International Conference on Cyberworlds (CW) (IEEE), 102-109. doi:10.1109/cw49994.2020.00022

Winkler, S., Kazazi, J., and Vollrath, M. (2018). How to Warn Drivers in Various Safety-Critical Situations - Different Strategies, Different Reactions. Accid. Anal. Prev. 117, 410-426. doi:10.1016/j.aap.2018.01.040

Wintersberger, P., Frison, A.-K., Riener, A., and Sawitzky, T. v. (2019). Fostering User Acceptance and Trust in Fully Automated Vehicles: Evaluating the Potential of Augmented Reality. PRESENCE: Virtual and Augmented Reality 27, 46-62. doi:10.1162/pres_a_00320

Wintersberger, P., Sawitzky, T. v., Frison, A.-K., and Riener, A. (2017). "Traffic Augmentation as a Means to Increase Trust in Automated Driving Systems," in Proceedings of the 12th biannual conference on italian sigchi, 1-7. doi:10.1145/ 3125571.3125600

Wu, H., Ashmead, D. H., Adams, H., and Bodenheimer, B. (2018a). "3D Sound Rendering in a Virtual Environment to Evaluate Pedestrian Street Crossing 
Decisions at a Roundabout," in 2018 IEEE 4th VR Workshop on Sonic Interactions for Virtual Environments (SIVE) (IEEE), 1-6. doi:10.1109/ sive.2018.8577195

Wu, H., Ashmead, D. H., and Bodenheimer, B. (2009). "Using Immersive Virtual Reality to Evaluate Pedestrian Street Crossing Decisions at a Roundabout," in Proceedings of the 6th Symposium on Applied Perception in Graphics and Visualization, 35-40. doi:10.1145/1620993.1621001

Wu, X., Merenda, C., Misu, T., Tanous, K., Suga, C., and Gabbard, J. L. (2020). “Drivers' Attitudes and Perceptions towards A Driving Automation System with Augmented Reality Human-Machine Interfaces," in 2020 IEEE Intelligent Vehicles Symposium (IV) (IEEE), 1978-1983. doi:10.1109/ iv47402.2020.9304717

Wu, Y., Abdel-Aty, M., Park, J., and Zhu, J. (2018b). Effects of Crash Warning Systems on Rear-End Crash Avoidance Behavior under Fog Conditions. Transportation Res. C: Emerging Tech. 95, 481-492. doi:10.1016/ j.trc.2018.08.001

Wulf, F., Rimini-Doring, M., Arnon, M., and Gauterin, F. (2015). Recommendations Supporting Situation Awareness in Partially Automated Driver Assistance Systems. IEEE Trans. Intell. Transport. Syst. 16, 2290-2296. doi:10.1109/tits.2014.2376572

$\mathrm{Xu}, \mathrm{Z}$., Zou, X., Oh, T., and Vu, H. L. (2020). Studying Freeway Merging Conflicts Using Virtual Reality Technology. J. Saf. Res.. doi:10.1016/ j.jsr.2020.11.002

Yang, B., Zheng, R., Shimono, K., Kaizuka, T., and Nakano, K. (2017). Evaluation of the Effects of In-vehicle Traffic Lights on Driving Performances for Unsignalised Intersections. IET Intell. Transport Syst. 11, 76-83. doi:10.1049/iet-its.2016.0084

Yang, C.-W., Lee, T.-H., Huang, C.-L., and Hsu, K.-S. (2016). "Unity 3D Production and Environmental Perception Vehicle Simulation Platform," in 2016 International Conference on Advanced Materials for Science and Engineering (ICAMSE)IEEE)), 452-455. doi:10.1109/ icamse.2016.7840349

Ye, Y., Wong, S. C., Li, Y. C., and Lau, Y. K. (2020). Risks to Pedestrians in Traffic Systems with Unfamiliar Driving Rules: a Virtual Reality Approach. Accid. Anal. Prev. 142, 105565. doi:10.1016/j.aap.2020.105565

Yeo, D., Kim, G., and Kim, S. (2020). “Toward Immersive Self-Driving Simulations: Reports from a User Study across Six Platforms," in Proceedings of the 2020
CHI Conference on Human Factors in Computing Systems, 1-12. doi:10.1145/ 3313831.3376787

Ying, Y., and Yuhui, Z. (2011). "Virtual Simulation Experiment Analysis of Chevron Deceleration Marking Based on Driving Simulator," in 2011 Fourth International Conference on Intelligent Computation Technology and Automation (IEEE), 1. 991-994. doi:10.1109/icicta.2011.249

Yu, Y., El Kamel, A., Gong, G., and Li, F. (2014). Multi-agent Based Modeling and Simulation of Microscopic Traffic in Virtual Reality System. Simulation Model. Pract. Theor. 45, 62-79. doi:10.1016/j.simpat.2014.04.001

Yu, Y., Kamel, A. E., and Gong, G. (2013). "Modeling Overtaking Behavior in Virtual Reality Traffic Simulation System," in 2013 9th Asian Control Conference (ASCC) (IEEE), 1-6. doi:10.1109/ascc.2013.6606247

Yun, H., and Yang, J. H. (2020). Multimodal Warning Design for Take-Over Request in Conditionally Automated Driving. Eur. Transp. Res. Rev. 12, 34. doi:10.1186/s12544-020-00427-5

Zimmermann, M., Schopf, D., Lütteken, N., Liu, Z., Storost, K., Baumann, M., et al. (2018). Carrot and Stick: A Game-Theoretic Approach to Motivate Cooperative Driving through Social Interaction. Transportation Res. Part C: Emerging Tech. 88, 159-175. doi:10.1016/j.trc.2018.01.017

Conflict of Interest: The authors declare that the research was conducted in the absence of any commercial or financial relationships that could be construed as a potential conflict of interest.

Publisher's Note: All claims expressed in this article are solely those of the authors and do not necessarily represent those of their affiliated organizations, or those of the publisher, the editors and the reviewers. Any product that may be evaluated in this article, or claim that may be made by its manufacturer, is not guaranteed or endorsed by the publisher.

Copyright (c) 2021 Riegler, Riener and Holzmann. This is an open-access article distributed under the terms of the Creative Commons Attribution License (CC BY). The use, distribution or reproduction in other forums is permitted, provided the original author(s) and the copyright owner(s) are credited and that the original publication in this journal is cited, in accordance with accepted academic practice. No use, distribution or reproduction is permitted which does not comply with these terms. 